\title{
Linearization instability for generic gravity in AdS spacetime
}

\author{
Emel Altas and Bayram Tekin \\ Department of Physics, Middle East Technical University, 06800 Ankara, Turkey
}

(Received 7 July 2017; published 24 January 2018)

\begin{abstract}
In general relativity, perturbation theory about a background solution fails if the background spacetime has a Killing symmetry and a compact spacelike Cauchy surface. This failure, dubbed as linearization instability, shows itself as non-integrability of the perturbative infinitesimal deformation to a finite deformation of the background. Namely, the linearized field equations have spurious solutions which cannot be obtained from the linearization of exact solutions. In practice, one can show the failure of the linear perturbation theory by showing that a certain quadratic (integral) constraint on the linearized solutions is not satisfied. For non-compact Cauchy surfaces, the situation is different and for example, Minkowski space having a non-compact Cauchy surface, is linearization stable. Here we study, the linearization instability in generic metric theories of gravity where Einstein's theory is modified with additional curvature terms. We show that, unlike the case of general relativity, for modified theories even in the non-compact Cauchy surface cases, there are some theories which show linearization instability about their anti-de Sitter backgrounds. Recent $D$ dimensional critical and three dimensional chiral gravity theories are two such examples. This observation sheds light on the paradoxical behavior of vanishing conserved charges (mass, angular momenta) for non-vacuum solutions, such as black holes, in these theories.
\end{abstract}

DOI: 10.1103/PhysRevD.97.024028

\section{INTRODUCTION}

There is a very interesting conundrum in nonlinear theories, such as Einstein's gravity or its modifications with higher curvature terms: exact solutions without symmetries (which are physically interesting) are hard to find, hence one resorts to symmetric "background" solutions and develops a perturbative expansion about them. But it turns out that exactly at the symmetric solutions, namely about solutions having Killing vector fields, naive first order perturbation theory fails under certain conditions. The set of solutions to Einstein's equations forms a smooth manifold except at the solutions with infinitesimal symmetries and spacetimes with compact Cauchy surfaces where there arise conical singularities in the solution space. Namely, perturbation theory in nonlinear theories can yield results which are simply wrong in the sense that some perturbative solutions cannot be obtained from the linearization of exact solutions. Roughly speaking, the process of first linearizing the field equations and then finding the solutions to those linearized equations; and the process of linearization of exact solutions to the nonlinear equations can yield different results if certain necessary criteria are

Published by the American Physical Society under the terms of the Creative Commons Attribution 4.0 International license. Further distribution of this work must maintain attribution to the author(s) and the published article's title, journal citation, and DOI. Funded by SCOAP ${ }^{3}$. not met with regard to the background solution about which perturbation theory is carried out. Actually, the situation is more serious: linearized field equations can have spurious solutions which do not come from exact solutions. This could happen for various reasons and the failure of the first order perturbation theory can be precisely defined, as we shall do below. Figure 1 summarizes the results.

Let us give a couple of early observations in this issue in the context of general relativity (GR) before we start the discussion in generic gravity. One clear way to see the failure of the perturbation theory is through the initial value formulation of the theory for globally hyperbolic, oriented,

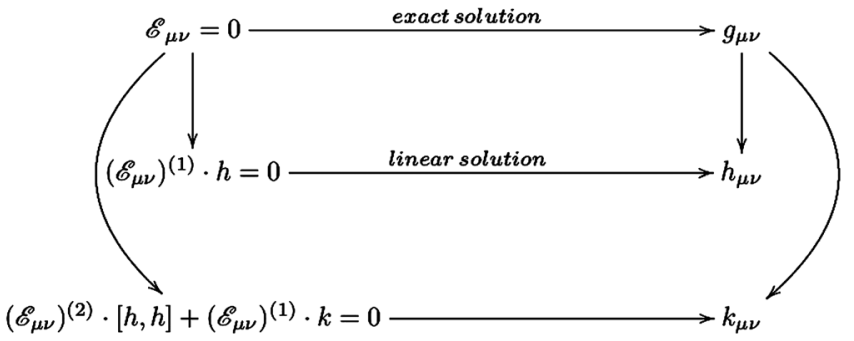

FIG. 1. The vertical straight arrows show first order linearization while the curved ones show second order linearization. For a linearization stable theory, the diagram makes sense and the solution to the linearized equation $h$ is not further restricted at the second order which means that there is a symmetric tensor $k$ that satisfies the second order equation in the bottom left. The details of the symbols are explained in the next section. 
time-orientable spacetimes with the topology $\mathcal{M} \approx \Sigma \times \mathbb{R}$, where $\Sigma$ is a spacelike Cauchy surface on which the induced Riemannian metric $\gamma$ and the extrinsic curvature $K$ (as well as matter content of the theory) are defined. ${ }^{1}$ We shall consider the matter-free case throughout the paper. Since GR is nonlinear, the initial data cannot be arbitrarily prescribed: they must satisfy the so called Hamiltonian and momentum constraints $\Phi_{i}(\gamma, K)=0$ with $i \in\{1,2,3,4\}$ in four dimensions. If a given initial data $(\bar{\gamma}, \bar{K})$ solving the constraints is not isolated, meaning the linearized constraint equations $\delta \Phi_{i}(\bar{\gamma}, \bar{K}) \cdot[\delta \gamma, \delta K]=0$ allow viable linearized solutions $(\delta \gamma, \delta K)$, then the theory is said to be linearization stable about the initial Cauchy data. Deser and Brill [1] showed that in GR with a compact Cauchy surface having the topology of a 3-torus, there are strong constraints on the perturbations of the initial data. Any such perturbation leads to contradictions in the sense that bulk integrals of conserved mass and angular momenta do not vanish, while since there is no boundary, they must vanish in this compact space: hence the background is an isolated solution. Put in another way, the linearized field equations about the background have solutions which do not come from the linearization of exact solutions. This happens because, as we shall see below, the linearized equations of the theory are not sufficient to constrain the linearized solutions: quadratic constraints on the linearized solutions, in the form of an integral (so called Taub conserved quantity first introduced in [2] for each Killing vector field), arise.

Most of the work regarding the linearization stability or instability in gravity has been in the context of GR with or without matter and with compact or with noncompact Cauchy surfaces. A nice detailed account of all these in the context of GR is given in the book [3] See also [4] where a chapter is devoted to this issue and the Taub conserved quantity construction which is not widely known in the physics community. Our goal here is to extend the discussion to generic gravity theories: we show that if the field equations of the theory are defined by the Einstein tensor plus a covariantly conserved two tensor, then a new source of linearization instability that does not exist in GR arises, especially in de Sitter or Anti-de Sitter backgrounds, with noncompact Cauchy surfaces. This happens because in these backgrounds there are special critical points in the space of parameters of the theory which conspire to cancel the conserved charge (mass, angular momentum etc) of nonperturbative objects (black holes) or the energies of the perturbative excitations. One needs to understand the origin of this rather interesting phenomenon that nonvacuum objects have the same charges as the vacuum. To give an example of this phenomenon let us note that this is

\footnotetext{
${ }^{1}$ It is also common to formulate the constraint equations in terms of $\gamma$ and a tensor density of weight 1 defined as $\pi:=$ $\sqrt{\operatorname{det} \gamma}\left(K-\gamma \operatorname{tr}_{\gamma} K\right)$ which is the conjugate momentum of the induced metric $\gamma$.
}

exactly what happens in chiral gravity [5-8] in $2+1$ dimensions where the Einstein tensor is augmented with the Cotton tensor and the cosmological constant times the metric (namely a special limit of the cosmological topologically massive gravity [9]). In AdS, at the chiral point, the contribution of the Cotton tensor and the Einstein tensor in AdS cancel each other at the level of the conserved charges. Exactly at that point, new ghostlike solutions, the so-called log modes arise [10] and if the boundary conditions are not those of Brown-Henneaux type [11], then these modes are present in the theory with negative energies. This would mean that the theory has no vacuum. But it was argued in $[6,8]$ that chiral gravity in AdS has a linearization instability which would remedy this problem. A similar phenomenon occurs in critical gravity in all dimensions $[12,13]$. Here we give a systematic discussion of the linearization stability and instability in generic gravity theories and study these two theories as examples. We will not follow the route of defining the theory in the $3+1$ setting and considering the instability problem on the Cauchy data. The reason for this is the following: in GR for asymptotically flat spacetimes, splitting the problem into the constraints on the Cauchy data and the evolution of the 3-metric and the extrinsic curvature turns the stability problem to a problem in elliptic operator theory which is well-developed and sufficient to rigorously prove the desired results. In the initial value formulation setting, the problem becomes a problem of determining the surjectivity of a linear operator, namely the linearized constraint operator. But this method is not convenient for our purposes since the source of the linearization instability in the extended gravity models that we shall discuss is quite different and so the full spacetime formulation is much better-suited for our problem. In GR as noted in the abstract, what saves the Minkowski space from the linearization instability is its noncompact Cauchy surfaces as was shown by Choquet-Bruhat and Deser [14]. This result is certainly consistent with the nonzero conserved charges (ADM mass or angular momentum) that can be assigned to an asymptotically-flat 3 dimensional Cauchy surface.

The layout of the paper is as follows: In Sec. II, we discuss the linearization stability in generic gravity theory and derive the second order constraints on the solutions of the linearized field equations. Of course these constraints are all related to the diffeomorphism invariance and the Bianchi identities of the theory. Hence we give a careful discussion of the linearized forms of the field equations and their gauge invariance properties. As the second order perturbation theory about a generic background is quite cumbersome in the local coordinates, we carry out the index-free computations in the bulk of the paper and relegate some parts of the component-wise computations to the appendices. In Sec. II, we establish the relation between the Taub conserved quantities coming from the second order perturbation theory and the Abbott- 
Deser-Tekin (ADT) charges coming from the first order perturbation theory. We study the linearization stability and instability of the Minkowski space, chiral gravity and critical gravity as examples. In a forthcoming paper, we shall give a more detailed analysis of the chiral gravity discussion in the initial value formulation context.

\section{LINEARIZATION STABILITY IN GENERIC GRAVITY}

Let us consider the matter-free equation of a generic gravity theory in an $D$-dimensional spacetime, whose dynamical field is the metric tensor $g$ only. In the indexfree notation the covariant two-tensor equation reads

$$
\mathcal{E}(g)=0,
$$

together with the covariant divergence condition which comes from the diffeomorphism invariance of the theory

$$
\delta_{g} \mathcal{E}(g)=0,
$$

where $\delta_{g}$ denotes the divergence operator with respect to the metric $g$. (As usual, one uses the musical isomorphism to extend the divergence from the contravariant tensors to the covariant ones.) Here we generalize the discussion in $[15,16]$ given for Einstein's theory to generic gravity. Let us assume that there is a one-parameter family of solutions to (1) denoted as $g(\lambda)$ which is at least twice differentiable with respect to $\lambda$ parametrizing the solution set. Then we can explore the consequences of this assumption with the help of the following identifications:

$\bar{g}:=\left.g(\lambda)\right|_{\lambda=0}, \quad h:=\left.\frac{d}{d \lambda} g(\lambda)\right|_{\lambda=0}, \quad k:=\left.\frac{d^{2}}{d \lambda^{2}} g(\lambda)\right|_{\lambda=0}$.

At this stage there is of course no immediate relation between the two covariant tensor fields $h$ (the first derivative of the metric) and $k$ (the second derivative of the metric) but, as we shall see later, consistency of the theory, i.e. the first order linearized and second order linearized forms of the field equations will relate them. We would first like to find that relation.

We assume that $\bar{g}$ exactly solves the vacuum equations $\mathcal{E}(\bar{g})=0$ and we compute the first derivative of the field equations with respect to $\lambda$ and evaluate it at $\lambda=0$ as

$$
\left.\frac{d}{d \lambda} \mathcal{E}(g(\lambda))\right|_{\lambda=0}=\left.D \mathcal{E}(g(\lambda)) \cdot \frac{d g(\lambda)}{d \lambda}\right|_{\lambda=0}=0
$$

where $D$ denotes the Fréchet derivative and the center-dot denotes "along the direction of the tensor that comes next" and we have used the chain rule. In local coordinates, this equation is just the first order "linearization" of the field equations (1) which we shall denote as $\left(\mathcal{E}_{\mu \nu}\right)^{(1)} \cdot h=0$. It is important to understand that solutions of (4) yield all possible $h$ tensors (up to diffeomorphisms), which are tangent to the exact solution $g(\lambda)$ at $\lambda=0$ in the space of solutions. To understand if there are any further constraints on the linearized solutions $h$, let us consider the second derivative of the field equation with respect to $\lambda$ and evaluate it at $\lambda=0$ to arrive at

$$
\begin{aligned}
& \left.\frac{d^{2}}{d \lambda^{2}} \mathcal{E}(g(\lambda))\right|_{\lambda=0} \\
& \quad=\left.\left(D^{2} \mathcal{E}(g(\lambda)) \cdot\left[\frac{d g(\lambda)}{d \lambda}, \frac{d g(\lambda)}{d \lambda}\right]+D \mathcal{E}(g(\lambda)) \cdot \frac{d^{2} g(\lambda)}{d \lambda^{2}}\right)\right|_{\lambda=0} \\
& \quad=0
\end{aligned}
$$

where we have used the common notation for the second Fréchet derivative in the first term and employed the chain rule when needed. We can write (5) in local coordinates as

$$
\left(\mathcal{E}_{\mu \nu}\right)^{(2)} \cdot[h, h]+\left(\mathcal{E}_{\mu \nu}\right)^{(1)} \cdot k=0,
$$

where again $\left(\mathcal{E}_{\mu \nu}\right)^{(2)} \cdot[h, h]$ denotes the second order linearization of the field equation about the background $\bar{g}$. Even though this equation is rather simple, it is important to understand its meaning to appreciate the rest of the discussion. This is the equation given in the bottom-left corner of Fig. 1. Given a solution $h$ of $\left(\mathcal{E}_{\mu \nu}\right)^{(1)} \cdot h=0$, Eq. (6) determines the tensor field $k$, which is the second order derivative of the metric $g(\lambda)$ at $\lambda=0$. If such a $k$ can be found then there is no further constraint on the linearized solution $h$. In that case, the field equations are said to be linearization stable at the exact solution $\bar{g}$. This says that the infinitesimal deformation $h$ is tangent to a full (exact) solution and hence it is integrable to a full solution. Of course, what is tacitly assumed here is that in solving for $k$ in (6), one cannot change the first order solution $h$, it must be kept intact for the perturbation theory to make any sense.

We can understand these results form a more geometric vantage point as follows. For the spacetime manifold $\mathcal{M}$, let $\mathcal{S}$ denote the set of solutions of the field equations $\mathcal{E}(g)=0$. The obvious question is (in a suitable Sobolev topology), when does this set of solutions form a smooth manifold whose tangent space at some "point" $\bar{g}$ is the space of solutions $(h)$ to the linearized equations? The folklore in the physics literature is not to worry about this question and just assume that the perturbation theory makes sense and the linearized solution can be improved to get better solutions, or the linearized solution is assumed to be integrable to a full solution. But as we have given examples above, there are cases when the perturbation theory fails and the set $\mathcal{S}$ has a conical singularity instead of being a smooth manifold. One should not confuse this situation with the case of dynamical instability as the latter really allows a "motion" or perturbation about a given solution. Here linearization instability refers to a literal breakdown of the first order perturbation theory. It is somewhat a 
nontrivial matter to show that there are no further constraints beyond the second order perturbation theory: In Einstein's gravity, this is related to the fact that constraint equations are related to zeros of the moment maps [17]. For generic gravity, this issue deserves to be further studied.

\section{A. Taub conserved quantities and ADT charges}

So far, in our discussion we have not assumed anything about whether the spacetime has a compact Cauchy surface or not. First, let us now assume that the spacetime has a compact spacelike Cauchy surface and has at least one Killing vector field. Then we can get an integral constraint on $h$, without referring to the $k$ tensor as follows. Let $\bar{\xi}$ be a Killing vector field of the metric $\bar{g}$, then the following vector field ${ }^{2}$

$$
T:=\bar{\xi} \cdot D^{2} \mathcal{E}(\bar{g}) \cdot[h, h],
$$

is divergence free, since $\delta_{\bar{g}} D^{2} \mathcal{E}(\bar{g}) \cdot[h, h]=0$ due to the linearized Bianchi identity. Then we can integrate $T$ over a compact hypersurface $\Sigma$ and observe that the integral (for the sake of definiteness, here we consider the $3+1$ dimensional case)

$$
\int_{\Sigma} d^{3} \Sigma \sqrt{\gamma} T \cdot \hat{n}_{\Sigma}
$$

is independent of hypersurface $\Sigma$ where $\gamma$ is the pull-back metric on the hypersurface and $\hat{n}_{\Sigma}$ is the unit future pointing normal vector. Let us restate the result in a form that we shall use below: given two compact disjoint hypersurfaces $\Sigma_{1}$ and $\Sigma_{2}$ in the spacetime $\mathcal{M}$, we have the statement of the "charge conservation" as the equality of the integration over the two hypersurfaces

$$
\int_{\Sigma_{1}} d^{3} \Sigma_{1} \sqrt{\gamma_{\Sigma_{1}}} T \cdot \hat{n}_{\Sigma_{1}}=\int_{\Sigma_{2}} d^{3} \Sigma_{2} \sqrt{\gamma_{\Sigma_{2}}} T \cdot \hat{n}_{\Sigma_{2}} \cdot
$$

We can now go to (6) and after contracting it with the Killing tensor $\bar{\xi}$, and integrating over $\Sigma$, we obtain the identity

$$
\int_{\Sigma} d^{3} \Sigma \sqrt{\gamma} \bar{\xi}^{\mu} \hat{n}^{\nu}\left(\mathcal{E}_{\mu \nu}\right)^{(2)} \cdot[h, h]=-\int_{\Sigma} d^{3} \Sigma \sqrt{\gamma} \bar{\xi}^{\mu} \hat{n}^{\nu}\left(\mathcal{E}_{\mu \nu}\right)^{(1)} \cdot k
$$

Let us study the right-hand side more carefully. In a generic theory, this conserved Killing charge is called the AbbottDeser-Tekin (ADT) charge when the symmetric two-tensor $k$ is the just the linearized two tensor $h[18,19]$. Once the

\footnotetext{
${ }^{2}$ For the lack of a better notation, note that $\bar{\xi}$ is contracted with the covariant background tensor with a center dot which we shall employ in what follows and it should not be confused with the center dot in the Fréchet derivative.
}

field equations of the theory are given, it is possible, albeit after some lengthy computation, to show that one can write the integral on the right-hand side as a total derivative.

$$
\bar{\xi}^{\mu}\left(\mathcal{E}_{\mu \nu}\right)^{(1)} \cdot h=\bar{\nabla}_{\alpha}\left(\mathcal{F}^{\alpha}{ }_{\nu \mu} \bar{\xi}^{\mu}\right),
$$

with an antisymmetric tensor $\mathcal{F}$ in $\alpha$ and $\nu$. Hence if the Cauchy surface is compact without a boundary, the ADT charge vanishes identically, namely

$$
Q_{\mathrm{ADT}}[\bar{\xi}]:=\int_{\Sigma} d^{3} \Sigma \sqrt{\gamma} \hat{n}^{\nu} \bar{\xi}^{\mu}\left(\mathcal{E}_{\mu \nu}\right)^{(1)} \cdot h=0,
$$

which via (10) says that one has the vanishing of the integral on the left-hand side which is called the Taub conserved quantity:

$$
Q_{\text {Taub }}[\bar{\xi}]:=\int_{\Sigma} d^{3} \Sigma \sqrt{\gamma} \hat{n}^{\nu} \bar{\xi}^{\mu}\left(\mathcal{E}_{\mu \nu}\right)^{(2)} \cdot[h, h]=0,
$$

which must be automatically satisfied for the case when $h$ is an integrable deformation. Otherwise this equation is a second order constraint on the linearized solutions. Even though the ADT potential $\mathcal{F}$ was explicitly found for a large family of gravity theories, such as Einstein's gravity [18], quadratic gravity [19], $f($ Riem $)$ theories [20], and some examples will be given below, we can still refine the above argument of the vanishing of both the ADT and Taub conserved quantities without referring to the ADT potential [or more explicitly without referring to (11)]. The following argument was given for Einstein's gravity in [16] which immediately generalizes to the most general gravity as follows: consider the ADT charge (12) and assume that in the spacetime one has two disjoint compact hypersurfaces $\Sigma_{1}$ and $\Sigma_{2}$ as above. Then the statement of conservation of the charge is simply

$$
Q_{\mathrm{ADT}}\left(\bar{\xi}, \Sigma_{1}\right)=Q_{\mathrm{ADT}}\left(\bar{\xi}, \Sigma_{2}\right) .
$$

Now let $k$ be a two tensor which is $k_{1}$ and nonzero on $\Sigma_{1}$ and $k_{2}$ and zero near $\Sigma_{2}$, then $Q_{\mathrm{ADT}}\left(\bar{\xi}, \Sigma_{2}\right)=0$ so $Q_{\mathrm{ADT}}\left(\bar{\xi}, \Sigma_{1}\right)=0$ which in turn yields the vanishing of the Taub conserved quantities via (10).

To summarize the results obtained so far, let us note that assuming an integrable infinitesimal deformation $h$, which is by definition a solution to the linearized field equations about a background $\bar{g}$ solution, we arrived at (6). And the discussion after that equation showed that Taub conserved quantities constructed with a Killing vector field, from the second order linearization, $\left(\mathcal{E}_{\mu \nu}\right)^{(2)} \cdot[h, h]$, and the ADT charges constructed from the first order linearization, $\left(\mathcal{E}_{\mu \nu}\right)^{(1)} \cdot h$, vanish identically for the case of compact Cauchy hypersurfaces without a boundary. If these integrals do not vanish, then there is a contradiction and the linearized solution $h$ is further constraint. Hence it is not an integrable deformation, namely, $h$ is not in the tangent 
space about the point $\bar{g}$ in the space of solutions. For Einstein's theory with compact Cauchy surfaces, it was shown that the necessary condition for linearization stability is the absence of Killing vector fields [21,22]. As noted above, the interesting issue is that further study reveals that besides the quadratic constraint, there are no other constraints on the solutions to the linearized equations [17].

\section{B. Gauge invariance of the charges}

Of course there is one major issue that we still must address that is the gauge-invariance (or coordinate independence) of the above construction which we show now. Following [16], first let us consider a (not necessarily small) diffeomorphism $\varphi$ of the spacetime as $\varphi: \mathcal{M} \rightarrow \mathcal{M}$. Then we demand that having obtained our rank two tensor $\mathcal{E}(g)$ from a diffeomorphism invariant action (or from a diffeomorphism invariant action up to a boundary term as in the case of topologically massive gravity) we have a global statement of diffeomorphism invariance as

$$
\mathcal{E}\left(\varphi^{*} g\right)=\varphi^{*} \mathcal{E}(g),
$$

which states that $\mathcal{E}$ evaluated for the pull-back metric is equivalent to the pull-back of $\mathcal{E}$ evaluated for $g$. Let us now consider a one-parameter family of diffeomorphisms as $\varphi_{\lambda}$, generated by a vector field $X$ well-defined on some region of spacetime. Let $\varphi_{0}$ be the identity diffeomorphism denoted as $\varphi_{0}=I_{\mathcal{M}}$. Then we can differentiate (15) with respect to $\lambda$ once to get

$$
\frac{d}{d \lambda} \mathcal{E}\left(\varphi_{\lambda}^{*} g\right)=\frac{d}{d \lambda} \varphi_{\lambda}^{*} \mathcal{E}(g),
$$

which, after making use of the chain rule, yields

$$
D \mathcal{E}\left(\varphi_{\lambda}^{*} g\right) \cdot \frac{d}{d \lambda} \varphi_{\lambda}^{*} g=\varphi_{\lambda}^{*}\left(\mathcal{L}_{X} \mathcal{E}(g)\right),
$$

where $\mathcal{L}_{X}$ is the Lie derivative with respect to the vector field $X$. Taking the derivative of the last equation with respect to $g$ yields

$$
D^{2} \mathcal{E}(g) \cdot\left(h, \mathcal{L}_{X} g\right)+D \mathcal{E}(g) \cdot \mathcal{L}_{X} h=\mathcal{L}_{X}(D \mathcal{E}(g) \cdot h) .
$$

In components, and after setting $\lambda=0$, Eq. (17) reads read, respectively

$$
\delta_{X}\left(\mathcal{E}_{\mu \nu}\right)^{(1)} \cdot h=\mathcal{L}_{X} \mathcal{E}_{\mu \nu}(\bar{g}),
$$

and Eq. (18) reads

$\delta_{X}\left(\mathcal{E}_{\mu \nu}\right)^{(2)} \cdot[h, h]+\left(\mathcal{E}_{\mu \nu}\right)^{(1)} \cdot \mathcal{L}_{X} h=\mathcal{L}_{X}\left(\mathcal{E}_{\mu \nu}\right)^{(1)} \cdot h$,

where $\delta_{X}\left(\mathcal{E}_{\mu \nu}\right)^{(1)} \cdot h$ denotes the variation of the background tensor $\left(\mathcal{E}_{\mu \nu}\right)^{(1)} \cdot h$ under the flow of $X$ or under the infinitesimal diffeomorphisms. Since $\mathcal{E}_{\mu \nu}(\bar{g})=0$, (19) says that $\left(\mathcal{E}_{\mu \nu}\right)^{(1)} \cdot h$ is gauge invariant: $\delta_{X}\left(\mathcal{E}_{\mu \nu}\right)^{(1)} \cdot h=0$. Similarly (20) yields

$$
\delta_{X}\left(\mathcal{E}_{\mu \nu}\right)^{(2)} \cdot[h, h]+\left(\mathcal{E}_{\mu \nu}\right)^{(1)} \cdot \mathcal{L}_{X} h=0,
$$

since $\left(\mathcal{E}_{\mu \nu}\right)^{(1)} \cdot h=0$ by assumption, the right-hand side of (20) vanishes. It is worth stressing that since generically $\left(\mathcal{E}_{\mu \nu}\right)^{(1)} \cdot \mathcal{L}_{X} h$ is not zero, the second order expansion $\left(\mathcal{E}_{\mu \nu}\right)^{(2)} \cdot[h, h]$ is not gauge invariant but transforms according to (21). Gauge invariance of the Taub conserved quantity and the ADT charge follows immediately from (21). Contracting that equation with the Killing vector field $\bar{\xi}$ and integrating over the Cauchy surface, one finds

$$
\int_{\Sigma} d^{3} \Sigma \sqrt{\gamma} n^{\nu}\left[\bar{\xi}^{\mu} \delta_{X}\left(\mathcal{E}_{\mu \nu}\right)^{(2)} \cdot[h, h]+\bar{\xi}^{\mu}\left(\mathcal{E}_{\mu \nu}\right)^{(1)} \cdot \mathcal{L}_{X} h\right]=0 .
$$

Since we have already shown that the second term can be written as a divergence we can drop it out, the remaining part is the Taub conserved quantity which is shown to be is gauge invariant, by this construction. The above discussion has been for a generic gravity theory based on the metric tensor as the only dynamical field, let us consider Einstein's gravity as an explicit example.

\section{Linearization stability in Einstein's gravity}

Let Ein denote the $(0,2)$ Einstein tensor, and $h$ denote a symmetric two tensor field as described above and $X$ be a vector field, then the effect of infinitesimal one-parameter diffeomorphisms generated by $X$ follows as

$$
D \operatorname{Ein}(g) \cdot \mathcal{L}_{X} g=\mathcal{L}_{X} \operatorname{Ein}(g)
$$

which in local coordinates reads

$$
\delta_{X}\left(G_{\mu \nu}\right)^{(1)} \cdot h=\mathcal{L}_{X} \bar{G}_{\mu \nu}
$$

where $G_{\mu \nu}:=\operatorname{Ein}\left(e_{\mu}, e_{\nu}\right)$ and $\operatorname{Ein}:=\operatorname{Ric}-\frac{1}{2} R g$. We have already given the proof of the above equation for a generic theory in the previous part, but it pays to do it more explicitly in Einstein's theory: so it follows as

$\delta_{X}\left(G_{\mu \nu}\right)^{(1)} \cdot h=\delta_{X}\left(R_{\mu \nu}\right)^{(1)} \cdot h-\frac{1}{2} \bar{g}_{\mu \nu} \delta_{X}(R)^{(1)} \cdot h-\frac{1}{2} \bar{R} \delta_{X} h_{\mu \nu}$,

which just comes from the definition of the linearized Einstein tensor. Then one can rewrite the above expression as desired: 


$$
\delta_{X}\left(G_{\mu \nu}\right)^{(1)} \cdot h=\mathcal{L}_{X}\left(\bar{R}_{\mu \nu}-\frac{1}{2} \bar{g}_{\mu \nu} \bar{R}\right)=\mathcal{L}_{X} \bar{G}_{\mu \nu}
$$

At the second order of linearization, one has

$$
D^{2} \operatorname{Ein}(g) \cdot\left(h, \mathcal{L}_{X} g\right)+D \operatorname{Ein}(g) \cdot \mathcal{L}_{X} h=\mathcal{L}_{X}(D \operatorname{Ein}(g) \cdot h),
$$

whose local version reads

$$
\delta_{X}\left(G_{\mu \nu}\right)^{(2)} \cdot[h, h]+\left(G_{\mu \nu}\right)^{(1)} \cdot \mathcal{L}_{X} h=\mathcal{L}_{X}\left(G_{\mu \nu}\right)^{(1)} \cdot h .
$$

The explicit proof of this expression is rather long, hence we relegate it to Appendix A.

Now let us study the linearization stability of a particular solution to Einstein's gravity with a cosmological constant. Let $\bar{g}$ solve the cosmological Einstein's field equations then the equation relevant to the study of linearization stability of this solution is (6) which now reads

$$
\left(\mathcal{G}_{\mu \nu}\right)^{(2)} \cdot[h, h]+\left(\mathcal{G}_{\mu \nu}\right)^{(1)} \cdot k=0,
$$

where $\left(\mathcal{G}_{\mu \nu}\right)^{(1)} \cdot k$ is a simple object but the second order object $\left(\mathcal{G}_{\mu \nu}\right)^{(2)} \cdot[h, h]$ is quite cumbersome. It is very hard to use this equation to show that for a generic background $\bar{g}_{\mu \nu}$, a $k_{\mu \nu}$ can be found or cannot be found that satisfy (29). Therefore one actually resorts to a weaker (sufficiency) condition that the Taub charges vanish which, as we have seen, results from integrating this equation after contracting with a Killing vector field $\xi^{\mu}$. To set the stage for generic gravity theories about their AdS backgrounds, let us study (29) in AdS and flat spaces. In that case one can plug an explicit ansatz as follows: assume that such a $k$ exists in the form

$$
k_{\mu \nu}=a h_{\mu \beta} h_{\nu}^{\beta}+b h h_{\mu \nu}+\bar{g}_{\mu \nu}\left(c h_{\alpha \beta}^{2}+d h^{2}\right),
$$

where $k:=k_{\mu \nu} \bar{g}^{\mu \nu}$ and $a, b, c, d$ are constants to be determined and all the raising and lowering is done with the background AdS metric $\bar{g}$. Here we shall work in $D$ spacetime dimensions. Inserting $k_{\mu \nu}$ as given in (30) in $\left(\mathcal{G}_{\mu \nu}\right)^{(1)} \cdot k$, and choosing $a=1$ and $b=-\frac{1}{2}$, one arrives at

$$
\left(\mathcal{G}_{\mu \nu}\right)^{(2)} \cdot[h, h]+\left(\mathcal{G}_{\mu \nu}\right)^{(1)} \cdot k=: K_{\mu \nu},
$$

where $K_{\mu \nu}$ is a tensor which must vanish if the background is linearization stable. Its explicit form is worked out in Appendix B. Let us consider the transverse traceless gauge, and make use of the field equations and the linearized field equations: Namely let us use $\bar{R}_{\mu \nu}=$ $\frac{2 \Lambda}{D-2} \bar{g}_{\mu \nu}$ and $\left(\mathcal{G}_{\mu \nu}\right)^{(1)} \cdot h=0$, which in this gauge reads $\square h_{\mu \nu}=\frac{4 \Lambda}{(D-1)(D-2)} h_{\mu \nu}$ to arrive at

$$
\begin{aligned}
K_{\mu \nu}= & \bar{\nabla}_{\alpha} H^{\alpha}{ }_{\mu \nu}+\frac{\Lambda}{D-2}\left(c(D-2)+\frac{1}{2}\right) \bar{g}_{\mu \nu} h_{\alpha \beta}^{2} \\
& -\frac{1}{4} \bar{\nabla}_{\nu} h^{\alpha \beta} \bar{\nabla}_{\mu} h_{\alpha \beta}-\frac{\Lambda D}{(D-1)(D-2)} h_{\mu \beta} h_{\nu}^{\beta},
\end{aligned}
$$

where the divergence piece is given as

$$
\begin{aligned}
H_{\mu \nu}^{\alpha}: & =\bar{\nabla}_{\alpha}\left[\frac { 1 } { 2 } \left(h^{\alpha \beta} \bar{\nabla}_{\beta} h_{\nu \mu}+h_{\beta \nu} \bar{\nabla}_{\mu} h^{\alpha \beta}+h_{\beta \mu} \bar{\nabla}_{\nu} h^{\alpha \beta}\right.\right. \\
& \left.\left.-h_{\mu \beta} \bar{\nabla}^{\alpha} h_{\nu}^{\beta}-h_{\mu \beta} \bar{\nabla}^{\beta} h_{\nu}^{\alpha}\right)\right] \\
& +\bar{\nabla}_{\alpha}\left[-\frac{1}{4} \bar{g}_{\mu \nu} h_{\sigma \beta} \bar{\nabla}^{\beta} h^{\sigma \alpha}+\left[c(2-D)-\frac{1}{2}\right] \delta_{\nu}^{\alpha} h^{\sigma \beta} \bar{\nabla}_{\mu} h_{\sigma \beta}\right. \\
& \left.+\left[c(D-2)+\frac{5}{8}\right] \bar{g}_{\mu \nu} h_{\sigma \beta} \bar{\nabla}^{\alpha} h^{\sigma \beta}\right] .
\end{aligned}
$$

In the transverse-traceless gauge, the coefficient $d$ is not fixed and can be set to zero. $K_{\mu \nu}$ has a single parameter $c$, that one can choose to fix the stability of the flat spacetime (which was proven by [14] using the linearization of the constraints on a noncompact Cauchy surface in Minkowski space). Before looking at the flat space case, let us note that one has $\bar{\nabla}_{\mu} K^{\mu \nu}=0$ as expected. Let us consider the flat space with $\Lambda=0$ and use the Cartesian coordinates so that $\bar{\nabla}_{\alpha} \rightarrow \partial_{\alpha}$. The corresponding linearized field equations become

$$
\partial^{2} h_{\mu \nu}=0
$$

together with the gauge choices $\partial_{\mu} h^{\mu \nu}=0=h$. The general solution of (33) can be exactly constructed as a superposition of plane-wave solutions, hence it suffices to study the linearized stability of flat space against the plane-wave modes which we take to be the real part of

$$
h_{\mu \nu}=\varepsilon_{\mu \nu} e^{i k \cdot x},
$$

together with $k^{\mu} \varepsilon_{\mu \nu}=0, \varepsilon_{\mu}^{\mu}=0$ and $k^{2}=0,{ }^{3}$ which follow from the gauge condition and (33). Evaluating $K_{\mu \nu}$ for this solution, one arrives at

$$
K_{\mu \nu}=k_{\nu} k_{\mu} \varepsilon_{\alpha \beta} \varepsilon^{\alpha \beta} e^{i k \cdot x}\left[2 c(D-2)+\frac{5}{4}\right],
$$

which vanishes for the choice

$$
c=-\frac{5}{8(D-2)}
$$

\footnotetext{
${ }^{3}$ In a compact space without a boundary, $k=0$ mode should also be considered, in that case one has the solution $h_{\mu \nu}=$ $\varepsilon_{\mu \nu}\left(c_{1} t+c_{2}\right)$ which gives rise to linearization instability [23] for the case of the torus.
} 
So (29) is satisfied for

$$
k_{\mu \nu}=h_{\mu \beta} h_{\nu}^{\beta}-\frac{5}{8(D-2)} \bar{g}_{\mu \nu} h_{\alpha \beta}^{2}
$$

and therefore there is no further constraint on the linearized solutions (34) and the Minkowski space is linearization stable. Next we move on to quadratic gravity theory.

\section{Linearization instability beyond Einstein's theory}

One of the reasons that lead us to study the linearization instability in generic gravity theories is an observation made in [19] where conserved charges of generic gravity theories for asymptotically AdS backgrounds were constructed. ${ }^{4}$ The observation was that in AdS backgrounds, the conserved energy and angular momenta vanish in generic gravity theories for all asymptotically AdS solutions at some particular values of the parameters defining the theory (in fact a whole section in that paper was devoted for the zero energy issue). This apparent infinite degeneracy of the vacuum for AdS spaces, is in sharp contrast to the flat space case where the unique zero energy is attained only by the Minkowski space, namely the classical ground state. Let us expound upon this a little more: for all purely metric based theories, the energy (mass) of the space-time that asymptotically approaches the flat space at spatial infinity is given by the ADM formula

$$
M_{\mathrm{ADM}}=\frac{1}{\kappa} \oint_{\partial \Sigma} d S_{i}\left(\partial_{j} h^{i j}-\partial^{i} h^{j}{ }_{j}\right) .
$$

It is well known that $M_{\mathrm{ADM}} \geq 0$, which is known as the positive energy theorem $[25,26]$. An important part of this theorem is that the vacuum, namely the flat space-time with $M_{\mathrm{ADM}}=0$, is unique (up to diffeomorphisms of course) $[27,28]$. It should be also noted that the ADM mass is defined in flat Cartesian coordinates but it was shown to be coordinate invariant. Here one must be very careful, if proper decaying conditions are not realized for $h_{i j}$, any (positive, negative, finite or divergent) value of mass can be assigned to the flat space. It is exactly these properties of the ADM formula that made it a useful tool in geometry: without even referring to Einstein's equations, one can take (38) to be a geometric invariant of an asymptotically flat manifold, modulo some decaying conditions on the first and the second fundamental forms of the spacelike surface.

Once one deviates from asymptotic flatness, then as we have noted, for higher derivative theories there are critical points which seem to make the vacuum infinitely degenerate, namely, the corresponding mass formula assigns any solution of the theory the same zero charge. Naively, one

\footnotetext{
${ }^{4}$ For an earlier zero energy result in the context of asymptotically flat backgrounds for purely quadratic gravity in four dimensions, see [24].
}

can try to understand the meaning of vanishing charges for nonvacuum solutions (namely, non-maximally symmetric solutions) as follows:

(i) There is a confinement of the relevant perturbations (in the weak coupling), just like in QCD in the strong coupling of color charge; and so a nonvacuum solution such as the proton has zero total color charge, same as the vacuum. In the case of QCD, perturbation theory might yield spurious states that cannot freely exist, such as quarks, as also noted in [6]. In gravity confinement would mean, confinement of massenergy or some other properties under consideration such as chirality. But this would be highly unphysical because if there are no other conserved charges to suppress the creation of confined mass, then the vacuum state of gravity would be infinitely degenerate and creating confined mass would cost nothing.

(ii) The second possibility is that perturbation theory about a given background solution, be it the maximally symmetric vacuum or not, may simply fail to exist just because the background solution is an isolated solution in the solution space. Namely, the solution space may fail to be a smooth manifold.

In fact, as discussed above, linearization of nonlinear equations such as Einstein's gravity and Yang-Mill's theory showed that naive first order perturbation theory fails generically when the background has a Killing symmetry. To be more specific we consider two recent examples: the chiral gravity in $2+1$ dimensions which is a special case of topologically massive gravity with a cosmological constant and the critical gravity which is a specific example of quadratic gravity in AdS. These examples can be easily extended, as the phenomenon we discuss is quite generic and take place whenever Einstein's theory with a cosmological constant is modified with some curvature terms.

To see how perturbation theory can fail let us go back to the necessary condition (6) and contract it with the Killing vector $\bar{\xi}^{\mu}$ to obtain

$$
\bar{\xi}^{\mu}\left(\mathcal{E}_{\mu \nu}\right)^{(2)} \cdot[h, h]+\bar{\xi}^{\mu}\left(\mathcal{E}_{\mu \nu}\right)^{(1)} \cdot k=0 .
$$

In some modified gravity theories one finds that the second term can be written as

$$
\bar{\xi}^{\mu}\left(\mathcal{E}_{\mu \nu}\right)^{(1)} \cdot k=c\left(\alpha_{i}, \bar{R}\right) \bar{\nabla}_{\alpha} \mathcal{F}_{1 \nu}^{\alpha}+\bar{\nabla}_{\alpha} \mathcal{F}_{2 \nu}^{\alpha},
$$

where $c\left(\alpha_{i}, \bar{R}\right)$ is a constant determined by the parameters $\alpha_{i}$ of the theory as well as the curvature invariants (symbolically denoted above as $\bar{R}$ ) of the background metric. $\mathcal{F}_{i}^{\alpha \nu}$ are antisymmetric background tensors. It turns out that for asymptotically AdS spacetimes $\mathcal{F}_{2}^{\alpha \nu}$ vanishes identically at the boundary as it involves higher derivative terms of the perturbation, while $\mathcal{F}_{1}^{\alpha \nu}$ need not if there are not so fast decaying fields such as for example the KerrAdS black holes. On the other hand for the particular choice 
of the parameters $c\left(\alpha_{i}, \bar{R}\right)=0$, one arrives at the constraint that again the Taub charges must vanish identically

$$
Q_{\text {Taub }}[\bar{\xi}]=\oint_{\Sigma} d^{D-1} \Sigma \sqrt{\gamma} \bar{\xi}^{\mu}\left(\mathcal{E}_{\mu \nu}\right)^{(2)} \cdot[h, h]=0 .
$$

But this time we have the additional nontrivial equation

$$
\oint_{\Sigma} d^{D-1} \Sigma \sqrt{\gamma} \bar{\xi}^{\mu}\left(\mathcal{E}_{\mu \nu}\right)^{(1)} \cdot h \neq 0 .
$$

In general it is very hard to satisfy these two conditions simultaneously for all solutions. Therefore some solutions to the linearized equations $h$ turn out to be not integrable to a full solution, hence the linearization instability of the AdS background in these critical theories. Let us stress that we have not assumed that the Cauchy surfaces are compact: this type of linearization instability arises even in the noncompact case.

\section{E. Linearization instability in quadratic gravity}

The message we would like to convey is a rather universal one in all generic higher derivative gravity theories, but for the sake of being concrete and yet sufficiently general, we shall consider the quadratic gravity theory with the action (in $D$ dimensions)

$$
\begin{aligned}
I= & \int d^{D} x \sqrt{-g}\left(\frac{1}{\kappa}\left(R-2 \Lambda_{0}\right)+\alpha R^{2}+\beta R_{\mu \nu}^{2}\right. \\
& \left.+\gamma\left(R_{\mu \nu \rho \sigma}^{2}-4 R_{\mu \nu}^{2}+R^{2}\right)\right),
\end{aligned}
$$

where the last term is organized into the Gauss-Bonnet form, which vanishes identically for $D=3$ and becomes a surface term for $D=4$. But for $D \geq 5$, it contributes to the field equations with at most second order derivatives in the metric, just like the Einstein-Hilbert part. Conserved gravitational charges of this theory in its asymptotically AdS backgrounds were constructed in [19] following the background space-time techniques developed in [18] which is an extension of the ADM approach [29]. For any theory with a Lagrangian density $\mathcal{L}=\frac{1}{\kappa}\left(R-2 \Lambda_{0}\right)+f\left(R_{\sigma \rho}^{\mu \nu}\right)$, for a generic differentiable function $f$ of the Riemann tensor an its contractions, the conserved charges follow from those of (43), as shown in [20] since any such theory can be written as a quadratic theory with effective coupling constants as far as its energy properties and particle content are concerned [30]. In what follows, we quote some of the computations done in [19] here to make the ensuing discussion complete. The field equations that follow from (43) are

$$
\begin{aligned}
\mathcal{E}_{\mu \nu}[g]= & \frac{1}{\kappa}\left(R_{\mu \nu}-\frac{1}{2} g_{\mu \nu} R\right)+2 \alpha R\left(R_{\mu \nu}-\frac{1}{4} g_{\mu \nu} R\right)+(2 \alpha+\beta)\left(g_{\mu \nu} \square-\nabla_{\mu} \nabla_{\nu}\right) R \\
& +2 \gamma\left\{R R_{\mu \nu}-2 R_{\mu \sigma \nu \rho} R^{\sigma \rho}+R_{\mu \sigma \rho \tau} R_{\nu}^{\sigma \rho \tau}-2 R_{\mu \sigma} R_{\nu}^{\sigma}-\frac{1}{4} g_{\mu \nu}\left(R_{\tau \lambda \rho \sigma}^{2}-4 R_{\sigma \rho}^{2}+R^{2}\right)\right\}+\beta \square\left(R_{\mu \nu}-\frac{1}{2} g_{\mu \nu} R\right) \\
& +2 \beta\left(R_{\mu \sigma \nu \rho}-\frac{1}{4} g_{\mu \nu} R_{\sigma \rho}\right) R^{\sigma \rho}=0 .
\end{aligned}
$$

As we shall study the stability/instability of the nonflat maximally symmetric solution (or solutions), let $\bar{g}$ represent such a solution with the curvature tensors normalized as

$$
\begin{aligned}
\bar{R}_{\mu \rho \nu \sigma} & =\frac{2 \Lambda}{(D-1)(D-2)}\left(\bar{g}_{\mu \nu} \bar{g}_{\rho \sigma}-\bar{g}_{\mu \sigma} \bar{g}_{\rho \nu}\right), \\
\bar{R}_{\mu \nu} & =\frac{2 \Lambda}{D-2} \bar{g}_{\mu \nu}, \quad \bar{R}=\frac{2 D \Lambda}{D-2} .
\end{aligned}
$$

The field equations reduce to a single quadratic equation:

$$
\begin{aligned}
& \frac{\Lambda-\Lambda_{0}}{2 \kappa}+k \Lambda^{2}=0, \\
& k \equiv(D \alpha+\beta) \frac{(D-4)}{(D-2)^{2}}+\gamma \frac{(D-3)(D-4)}{(D-1)(D-2)} .
\end{aligned}
$$

For generic values of the parameters of the theory, of course, there may not be real solution and so the theory may not posses a maximally symmetric vacuum, but here we assume that there is a real solution to this algebraic equation (so $8 \Lambda_{0} k \kappa+1 \geq 0$ ) and study the linearization stability of this solution, which we call the (classical) vacuum or the background. One can then linearize the field equations (44) about the vacuum and get at the linear order

$$
\begin{gathered}
c_{1}\left(\mathcal{G}_{\mu \nu}\right)^{(1)}+(2 \alpha+\beta)\left(\bar{g}_{\mu \nu} \square-\bar{\nabla}_{\mu} \bar{\nabla}_{\nu}+\frac{2 \Lambda}{D-2} \bar{g}_{\mu \nu}\right)(R)^{(1)} \\
+\beta\left(\bar{\square}\left(\mathcal{G}_{\mu \nu}\right)^{(1)}-\frac{2 \Lambda}{D-1} \bar{g}_{\mu \nu}(R)^{(1)}\right)=0,
\end{gathered}
$$

where the constant in front of the first term is

$$
c_{1} \equiv \frac{1}{\kappa}+\frac{4 \Lambda D}{D-2} \alpha+\frac{4 \Lambda}{D-1} \beta+\frac{4 \Lambda(D-3)(D-4)}{(D-1)(D-2)} \gamma,
$$


and the linearized (background) tensors read

$$
\left(\mathcal{G}_{\mu \nu}\right)^{(1)}=\left(R_{\mu \nu}\right)^{(1)}-\frac{1}{2} \bar{g}_{\mu \nu}(R)^{(1)}-\frac{2 \Lambda}{D-2} h_{\mu \nu},
$$

which is just the linearized cosmological Einstein's tensor given in terms of the linearized Ricci tensor and the linearized scalar curvature:

$$
\begin{aligned}
\left(R_{\mu \nu}\right)^{(1)} & =\frac{1}{2}\left(\bar{\nabla}^{\sigma} \bar{\nabla}_{\mu} h_{\nu \sigma}+\bar{\nabla}^{\sigma} \bar{\nabla}_{\nu} h_{\mu \sigma}-\square h_{\mu \nu}-\bar{\nabla}_{\mu} \bar{\nabla}_{\nu} h\right), \\
(R)^{(1)} & =-\bar{\square} h+\bar{\nabla}^{\sigma} \bar{\nabla}^{\mu} h_{\sigma \mu}-\frac{2 \Lambda}{D-2} h .
\end{aligned}
$$

Given a background Killing vector $\bar{\xi}$, (there are $D(D+1) / 2$ number of Killing vectors for this space and the arguments work for any one of these) if we had not truncated the expansion of the field equations at $\mathcal{O}(h)$ but collected all the nonlinear terms on the right-hand side, we would have gotten

$$
\bar{\xi}^{\mu}\left(\mathcal{E}_{\mu \nu}\right)^{(1)} \cdot h:=\bar{\xi}^{\mu} T_{\mu \nu}\left[h^{2}, h^{3}, \ldots h^{n} \ldots\right] .
$$

where $T_{\mu \nu}\left[h^{2}, h^{3}, \ldots h^{n} \ldots\right]$ represents all the higher order terms (and if there is a matter source with compact support of energy-momentum tensor, it also includes that). The next step is the crucial step: as was shown in [19], one can write (50) as a divergence of two pieces as described by (40)

$$
\bar{\xi}^{\mu}\left(\mathcal{E}_{\mu \nu}\right)^{(1)} \cdot h=c \bar{\nabla}_{\alpha} \mathcal{F}_{1 \nu}^{\alpha}+\bar{\nabla}_{\alpha} \mathcal{F}_{2 \nu}^{\alpha},
$$

where the constant $c_{1}$ given in (48) is shifted due to the $\beta$ term as

$$
c \equiv c_{1}+\frac{4 \Lambda}{(D-1)(D-2)} \beta .
$$

The explicit forms of the $\mathcal{F}_{i}^{\mu \rho}$ tensors are found to be

$$
\begin{aligned}
\mathcal{F}_{1}^{\mu \rho}= & 2 \bar{\xi}_{\nu} \bar{\nabla}^{[\mu} h^{\rho] \nu}+2 \bar{\xi}^{[\mu} \bar{\nabla}^{\rho]} h+2 h^{\nu[\mu} \bar{\nabla}^{\rho]} \bar{\xi}_{\nu} \\
& +2 \bar{\xi}^{[\rho} \bar{\nabla}_{\nu} h^{\mu] \nu}+h \bar{\nabla}^{\mu} \bar{\xi}^{\rho},
\end{aligned}
$$

and

$$
\begin{aligned}
\mathcal{F}_{2}^{\mu \rho}= & (2 \alpha+\beta)\left(2 \bar{\xi}^{[\mu} \bar{\nabla}^{\rho]}(R)^{(1)}+(R)^{(1)} \bar{\nabla}^{\mu} \bar{\xi}^{\rho}\right) \\
& +2 \beta\left(\bar{\xi} \sigma \bar{\nabla}^{[\rho}\left(\mathcal{G}^{\mu]}{ }_{\sigma}\right)^{(1)}+\left(\mathcal{G}^{[\rho \sigma}\right)^{(1)} \bar{\nabla}^{\mu]} \bar{\xi}_{\sigma}\right) .
\end{aligned}
$$

For asymptotically AdS spacetimes, $\mathcal{F}_{2}^{\mu \rho}$ vanishes at spatial infinity due to the vanishing of both of $(R)^{(1)}$ and $\left(\mathcal{G}_{\mu \sigma}\right)^{(1)}$. As discussed in the previous section, vanishing of the constant $c$ leads to two strong constraints (41) and (42) on the linearized solution $h$ which is a statement of the instability of the background solution. Note that, for this higher order theory, we have not assumed that the spatial hypersurface is compact. (In fact, to be more accurate, AdS is not globally hyperbolic and does not have a Cauchy surface but one can work in the double cover which does).

The point at which $c=0$ is the point when the mass of the spin-2 massive mode also vanishes and further, assuming $4 \alpha(D-1)+D \beta=0$, one can also decouple the massive spin-0 mode in this theory and arrive at the so called critical gravity defined in $D=4$ [12] for generic $D$ in [13]. All these conditions are compatible with the existence of a maximally symmetric vacuum. For critical gravity, the apparent mass and angular momenta of all black holes and perturbative excitations with asymptotically AdS conditions vanish. ${ }^{5}$ But as we have seen here, perturbation theory used for both the excitations and construction of conserved quantities does not work exactly at the critical point: namely, the theory for the AdS background is not linearization stable. At the chiral point, there arise exact log-modes in chiral gravity $[31,32]$ which are of the wave type but they do not correspond to the linearized log-modes of [10].

Just for the sake of completeness, let us note that if $c \neq 0$, then the perturbation theory makes sense and the conserved charges of the theory for any asymptotically AdS solutions (such as the Kerr-AdS black holes) are simply given in terms of the conserved charges of the same solution in Einstein's gravity as

$$
\frac{Q_{\text {quad }}(\bar{\xi})}{Q_{\text {Einstein }}(\bar{\xi})}=-\beta m_{g}^{2},
$$

where $m_{g}$ is the mass of the spin-2 graviton given as

$-\beta m_{g}^{2}=\frac{1}{\kappa}+\frac{4 \Lambda(D \alpha+\beta)}{D-2}+\frac{4 \Lambda(D-3)(D-4)}{(D-1)(D-2)} \gamma$.

In (55), $Q_{\text {Einstein }}(\bar{\xi})$ refers to (with $\kappa_{\text {Newton }}=1$ ) the conserved charge (mass, angular momenta) in the cosmological Einstein's theory.

\section{F. Linearization instability in chiral gravity}

A model of quantum gravity even in the simpler $2+1$ dimensional setting has been rather elusive. One of the latest promising proposals was the so called chiral gravity [5] which is a specific limit of topologically massive gravity (TMG) [9] with the asymptotically AdS boundary conditions. TMG, as opposed to Einstein's gravity has nontrivial local dynamics hence in this respect, it might be more relevant to the four dimensional gravity both at the classical and quantum level. The crux of the arguments of the quantum version chiral gravity is that the bulk theory is dual to a unitary and chiral conformal field theory (CFT) on the two dimensional boundary, whose symmetry is known

\footnotetext{
${ }^{5}$ The energy of the perturbative bulk excitations can be constructed using the Ostrogradsky Hamiltonian [13].
} 
to be one of the two copies of the Virasoro algebra [11]. Finding the correct conformal field theory would amount to defining the quantum gravity via the AdS/CFT duality [33]. But immediately after the proposal of chiral gravity, it was realized that the theory has arbitrarily negative energy $\log$ modes that appear exactly at the chiral point and not only the dual CFT is not unitary (but a logarithmic one), but apparently chiral gravity does not have even a classical vacuum [10]. If true, this of course would be disastrous for chiral gravity. But later it was argued in $[6,8]$ that chiral gravity has linearization instability against these log modes in AdS: namely, these perturbative negative energy solutions do not actually come from the linearization of any exact solution. If that is the case, then linearization instability saves chiral gravity certainly at the classical level and perhaps at the quantum level. Here we give further arguments of the existence of linearization instability in chiral gravity.

The field equations of topologically massive gravity [9] with a negative cosmological constant $\left(\Lambda:=-\frac{1}{\ell^{2}}\right)$ is

$$
R_{\mu \nu}-\frac{1}{2} g_{\mu \nu} R-\frac{1}{\ell^{2}} g_{\mu \nu}+\frac{1}{\mu} C_{\mu \nu}=0,
$$

where the Cotton tensor in terms of the antisymmetric tensor and the covariant derivative of the Schouten tensor reads

$$
C_{\mu \nu}=\eta_{\mu}{ }^{\alpha \beta} \nabla_{\alpha} S_{\beta \nu}, \quad S_{\mu \nu}=R_{\mu \nu}-\frac{1}{4} g_{\mu \nu} R .
$$

The boundary theory has two copies of the Virasoro algebra [11] for asymptotically AdS boundary conditions given as

$$
c_{R / L}=\frac{3 l}{2 G_{3}}\left(1 \pm \frac{1}{\mu l}\right),
$$

and the bulk theory has a single helicity 2 mode with a mass-square

$$
m_{g}^{2}=\mu^{2}-\frac{1}{l^{2}} .
$$

It was shown in [34] that the contraction of the Killing vector $(\bar{\xi})$ with the linearized equations coming from (57) yields

$\bar{\xi}^{\mu}\left(\left(\mathcal{G}_{\mu \nu}\right)^{(1)}+\frac{1}{\mu}\left(C_{\mu \nu}\right)^{(1)}\right)=\bar{\nabla}_{\alpha} \mathcal{F}_{1 \nu}^{\alpha}[\bar{\Xi}]+\bar{\nabla}_{\alpha} \mathcal{F}_{3 \nu}^{\alpha}[\bar{\xi}]$,

where $\mathcal{F}_{1}^{\mu \rho}$ was given in (53) whereas one finds $\mathcal{F}_{3}^{\mu \rho}$ to be $\mathcal{F}_{3}^{\mu \rho}[\bar{\xi}]=\eta^{\mu \rho \beta}\left(\mathcal{G}_{\nu \beta}\right)^{(1)} \bar{\xi}^{\nu}+\eta^{\nu \rho \beta}\left(\mathcal{G}^{\mu}{ }_{\beta}\right)^{(1)} \bar{\xi}_{\nu}+\eta^{\mu \nu \beta}\left(\mathcal{G}_{\beta}{ }_{\beta}\right)^{(1)} \bar{\xi}_{\nu}$, where a new (twisted) Killing vector (㐫) appears:

$$
\bar{\Xi}^{\alpha}:=\bar{\xi}^{\alpha}+\frac{1}{2 \mu} \eta^{\alpha \beta \nu} \bar{\nabla}_{\beta} \bar{\xi}_{\nu}
$$

The conserved charges of TMG for asymptotically AdS backgrounds read as an integral over the circle at infinity as

$$
Q[\bar{\xi}]=\frac{1}{8 \pi G_{3}} \oint_{\partial \mathcal{M}} d S_{i}\left(\mathcal{F}_{1}^{0 i}[\bar{\Xi}]+\frac{1}{2 \mu} \mathcal{F}_{3}^{0 i}[\bar{\xi}]\right) .
$$

Once again for the asymptotically $\operatorname{AdS}$ cases $\mathcal{F}_{3}^{\alpha}{ }_{\nu}[\bar{\xi}]$ vanishes identically on the boundary as it involves the linearized Einstein tensor at infinity. For generic values of $\mu$ and $\ell$, the first term, that is $\mathcal{F}_{1}^{\alpha}[\bar{\Xi}]$ gives the conserved charges for the corresponding Killing vector. But, for $\mu^{2} \ell^{2}=1$, as was shown in [35] the angular momentum and the energy of the rotating black hole solutions with the rotation parameter $(j)$ and the mass $(m)$ related as $(j=m \ell)$ (the extremal BTZ black hole) vanishes identically. This particular point was further studied in [5] where it was argued and conjectured that the theory, so called chiral gravity, as one of the central charges noted above (59) becomes zero, makes sense both classically and quantum mechanically.

Classically the theory should have a stable vacuum and quantum mechanically, it should have a dual healthy boundary conformal field theory. In [5] it was shown that all the bulk excitations have vanishing energy exactly at the chiral point. Later new log modes that were not accounted for were found in [10] which violated the existence of a ground state (namely, these modes have arbitrarily large negative energy compared to the zero energy of the vacuum). For further work on chiral gravity, see [36,37]. In $[6,8]$ it was argued that the AdS has linearization instability in chiral gravity against these log modes. Here, our construction lends support to these arguments.

For the sake of concreteness, let us consider the background metric as

$$
\bar{g}=-\left(1+\frac{r^{2}}{\ell^{2}}\right) d t^{2}+\frac{d r^{2}}{1+\frac{r^{2}}{\ell^{2}}}+r^{2} d \phi^{2},
$$

then for $\bar{\xi}=(-1,0,0)$ referring to the timelike energy Killing vector, one finds the twisted Killing vector to be

$$
\Xi=\left(-1,0,-\frac{1}{\ell^{2} \mu}\right)
$$

For this $\Xi$ to be a time-like Killing vector for all $r$ including the boundary at $r \rightarrow \infty$, one can see that (excluding the trivial $\mu \rightarrow \infty$ case) one must set $\mu^{2} \ell^{2}=1$, which is the chiral gravity limit. To further see this chiral gravity limit, let us recast $\mathcal{F}_{1}^{\mu \rho}[\Xi]$ using the superpotential $\mathcal{K}^{\mu \alpha \nu \beta}$ is defined by [18] 


$$
\begin{aligned}
\mathcal{K}^{\mu \nu \alpha \beta} & :=\frac{1}{2}\left(\bar{g}^{\mu \beta} \tilde{h}^{\nu \alpha}+\bar{g}^{\nu \alpha} \tilde{h}^{\mu \beta}-\bar{g}^{\mu \nu} \tilde{h}^{\alpha \beta}-\bar{g}^{\alpha \beta} \tilde{h}^{\mu \nu}\right), \\
\tilde{h}^{\mu \nu} & :=h^{\mu \nu}-\frac{1}{2} \bar{g}^{\mu \nu} h,
\end{aligned}
$$

which yields

$$
\mathcal{F}_{1}^{\mu \rho}[\Xi]=\Xi_{\nu} \bar{\nabla}_{\beta} \mathcal{K}^{\mu \rho \nu \beta}-\mathcal{K}^{\mu \sigma \nu \rho} \bar{\nabla}_{\sigma} \bar{\Xi}_{\nu}
$$

For all asymptotically AdS solutions with the BrownHenneaux boundary conditions, one can show that

$$
\mathcal{F}_{1}^{\mu \rho}[\Xi]=\left(1-\frac{1}{\ell^{2} \mu^{2}}\right) \mathcal{F}_{1}^{\mu \rho}[\bar{\xi}]
$$

which vanishes at the chiral point. So exactly at this point, there exists second order integral constraints on the linearized solutions as discussed in the previous section. The log-modes of [10] do not satisfy these integral constraints and so fail to be integrable to full solutions. ${ }^{6}$

Let us compute the value of the Taub conserved quantity for the log solution which was given in the background with the global coordinates for which the metric reads

$$
d s^{2}=\ell^{2}\left(-\cosh ^{2} \rho d \tau^{2}+\sinh ^{2} \rho d \phi^{2}+d \rho^{2}\right) .
$$

For the coordinates $u=\tau+\phi, v=\tau-\phi$, at exactly in the chiral point, one has the following additional solution

$$
\begin{aligned}
h_{\mu \nu}= & \frac{\sinh \rho}{\cosh ^{3} \rho}(\cos (2 u) \tau-\sin (2 u) \ln \cosh \rho)\left(\begin{array}{lll}
0 & 0 & 1 \\
0 & 0 & 1 \\
1 & 1 & 0
\end{array}\right)_{\mu \nu} \\
& -\tanh ^{2} \rho(\sin (2 u) \tau+\cos (2 u) \\
& \times \ln \cosh \rho)\left(\begin{array}{lll}
1 & 1 & 0 \\
1 & 1 & 0 \\
0 & 0 & -\sinh ^{-2} \rho \cosh ^{-2} \rho
\end{array}\right)_{\mu \nu} \cdot
\end{aligned}
$$

Considering the Killing vector $\bar{\xi}=(-1,0,0)$ one finds the result of the integral in (13) to be nonvanishing

$$
Q_{\text {Taub }}[\bar{\xi}]=\frac{\pi}{2 \ell}\left(3 \tau^{2}-\frac{161}{72}\right)
$$

which shows that this log mode is not in the tangent space of the solution space of chiral gravity around the $\mathrm{AdS}_{3}$ metric.

\footnotetext{
${ }^{6}$ See [38] a nice compilation of possible applications of logarithmic field theories in the context of holography and gravity.
}

\section{CONCLUSIONS AND DISCUSSIONS}

We have shown that at certain critical parameter values of extended gravity theories in constant curvature backgrounds, perturbation theory fails. Our arguments provide support to the discussion given by $[6,8]$ regarding the linearization instability in three dimensional chiral gravity and extend the discussion to generic gravity theories in a somewhat former form. The crucial point is that even in spacetimes with noncompact Cauchy surfaces, linearization instability can exist for background metrics with at least one Killing vector field. Our computation also sheds light on the earlier observations [19] that at certain critical values of the parameters defining the theory, conserved charges of all solutions, such as black holes, excitations vanish identically. ${ }^{7}$ For example, Kerr-AdS black hole metrics have the same mass and angular momentum as the AdS background. This leads to a rather nonphysical infinite degeneracy of the vacuum: for example, creating back holes costs nothing which is unacceptable. With our discussion above, it is now clear that, perturbation theory which is used to define boundary integrals of the conserved Killing charges does not make sense exactly at the critical values of the parameters. Therefore one really needs a new method to find/define conserved charges in these theories at their critical points. One such method was proposed in for quadratic theories [40] and in [41] for TMG.

We must note that, for asymptotically flat spacetimes, the ADM mass is the correct definition of mass-energy for any metric-based theory of gravity. Therefore, the stability of the Minkowski space as was shown for Einstein's theory by Choquet-Bruhat and Deser [14] is valid for all higher derivative models as long as one considers the noncompact Cauchy surfaces and asymptotically flat boundary conditions. But once a cosmological constant is introduced, the problem changes dramatically as we have shown: the ADM mass-energy (or angular momentum) expressions are modified and conserved charges get contributions from each covariant tensors added to the field equations. Once such a construction is understood, it is clear that some theories will have identically vanishing charges for all solutions with some fixed boundary conditions, which is a signal of linearization instability.

It is also important to realize that, linearization instability of certain background solutions in some theories is not bad as it sounds: for example chiral gravity is a candidate both as a nontrivial classical and quantum gravity theory in $\mathrm{AdS}_{3}$ with a two dimensional chiral conformal field theory induced on the boundary. But it has log-mode solutions which appear as ghosts in the classical theory and negative norm states in the quantum theory. It just turns out that chiral gravity in $\mathrm{AdS}_{3}$ has linearization instability along these log-modes: namely, they do not have vanishing Taub

\footnotetext{
${ }^{7}$ For a recent review of conserved charges in generic gravity theories see the book [39].
} 
conserved quantities which is a constraint for all integrable solutions. Therefore, they cannot come from linearization of exact solutions. A similar phenomenon takes place for the minimal massive gravity [42] which was proposed as a possible solution to the bulk-boundary unitarity clash in three dimensional gravity theories and as a viable model that has a healthy dual conformal field theory on the boundary of $\mathrm{AdS}_{3}$. It was shown recently in [43] that this theory only makes sense at the chiral point $[44,45]$ and hence linearization instability arises at that point which can save the theory from its log-modes. Let us note that we have also computed the second order constraint in the minimal massive gravity, namely the Taub conserved quantity and found that it is non-vanishing.

In the discussion of linearization stability and instability of a given exact solution in the context of general relativity, we noted that to make use of the powerful techniques of elliptic operator theory, on rewrites the four dimensional Einstein's theory as a dynamical system with constraints on a spacelike Cauchy surface and the evolution equations. As the constraints are intact, initial Cauchy data uniquely defines a spacetime (modulo some technical assumptions). Therefore, to study the linearization stability one can simply study the linearization stability of the constraints on the surface where the metric tensor field is positivedefinite. All these arguments boil down to showing that the initial background metric is not a singular point and that the space of solutions around the initial metric is an open subset (in fact a submanifold) of all solutions. This can be shown by proving the surjectivity of the operators that appear in the linearized constraints. A similar construction, dynamical formulation of the higher derivative models studied here in AdS and the surjectivity of the relevant linear maps would be highly valuable. For the case of the cosmological Einstein's theory, such a construction was carried out in [46] where it was observed that certain strong decays lead to linearization instability even for noncompact Cauchy surfaces with hyperbolic asymptotics.

\section{ACKNOWLEDGMENTS}

B. T. would like to thank S. Deser for extended discussions on conserved charges in generic gravity theories and on the meaning of vanishing charges. We would like to thank D. Grumiller for a useful exchange on the log-modes of chiral gravity.

\section{APPENDIX A: SECOND ORDER PERTURBATION THEORY AND GAUGE INVARIANCE ISSUES}

Here without going into too much detail let us summarize some of the relevant formulas that we use in the bulk of the paper to show various expressions, such as the gauge transformation of the background tensors, second order forms of the tensors, etc.
Lie and covariant derivatives do not commute so we shall need the following expressions. Let $X$ be a vector field on our manifold with a metric $\bar{g}$ and $T$ be a $(0,2)$ background tensor field. Then in components one has the Lie derivative of $T$ with respect to $X$ as

$$
\mathcal{L}_{X} T_{\rho \sigma}=X^{f} \bar{\nabla}_{f} T_{\rho \sigma}+\left(\bar{\nabla}_{\rho} X^{f}\right) T_{f \sigma}+\left(\bar{\nabla}_{\sigma} X^{f}\right) T_{\rho f} .
$$

Then one has the following difference of derivatives

$$
\begin{aligned}
\bar{\nabla}_{\mu} \mathcal{L}_{X} T_{\rho \sigma}-\mathcal{L}_{X} \bar{\nabla}_{\mu} T_{\rho \sigma}= & \left(\bar{\nabla}_{\mu} \bar{\nabla}_{\rho} X^{f}+X^{\lambda} \bar{R}_{\mu \lambda \rho}^{f}\right) T_{f \sigma} \\
& +\left(\bar{\nabla}_{\mu} \bar{\nabla}_{\sigma} X^{f}+X^{\lambda} \bar{R}_{\mu \lambda \sigma}{ }^{f}\right) T_{f \rho} .
\end{aligned}
$$

Let $\delta_{X}$ denote the gauge transformation generated by $X$, then the gauge transformation of the Christoffel connection reads,

$$
\delta_{X}\left(\Gamma_{\mu \nu}^{\gamma}\right)^{(1)}=\bar{\nabla}_{\mu} \bar{\nabla}_{\nu} X^{\gamma}+\bar{R}_{\nu \sigma \mu}^{\gamma} X^{\sigma} .
$$

Making use of this one finds

$$
\begin{aligned}
\bar{\nabla}_{\mu} \mathcal{L}_{X} T_{\rho \sigma}= & \mathcal{L}_{X} \bar{\nabla}_{\mu} T_{\rho \sigma}+T_{\alpha \sigma} \delta_{X}\left(\Gamma_{\mu \rho}{ }^{\alpha}\right)^{(1)} \\
& +T_{\rho \alpha} \delta_{X}\left(\Gamma_{\mu \sigma}{ }^{\alpha}\right)^{(1)}
\end{aligned}
$$

Applying the same procedure for the case of any generic three index tensor, we arrive the relation

$$
\begin{aligned}
\bar{\nabla}_{\mu} \mathcal{L}_{X} T_{\rho \sigma \gamma}= & \mathcal{L}_{X} \bar{\nabla}_{\mu} T_{\rho \sigma \gamma}+T_{\alpha \sigma \gamma} \delta_{X}\left(\Gamma_{\mu \rho}{ }^{\alpha}\right)^{(1)} \\
& +T_{\rho \alpha \gamma} \delta_{X}\left(\Gamma_{\mu \sigma}{ }^{\alpha}\right)^{(1)}+T_{\rho \sigma \alpha} \delta_{X}\left(\Gamma_{\mu \gamma}{ }^{\alpha}\right)^{(1)}
\end{aligned}
$$

Let us summarize some results about the second order perturbation theory (see also [47]) By definition one has

$$
g_{\mu \nu}:=\bar{g}_{\mu \nu}+\tau h_{\mu \nu},
$$

whose inverse is

$$
g^{\mu \nu}=\bar{g}^{\mu \nu}+\tau h^{\mu \nu}+\tau^{2} h_{\alpha}^{\mu} h^{\alpha \nu}+O\left(\tau^{3}\right) .
$$

Let $T$ be a generic tensor, then it can be expanded as

$$
T=\bar{T}+\tau T^{(1)}+\tau^{2} T^{(2)}+O\left(\tau^{3}\right) .
$$

For the Christoffel connection we have

$$
\Gamma_{\mu \nu}^{\gamma}=\bar{\Gamma}_{\mu \nu} \gamma+\tau\left(\Gamma_{\mu \nu} \gamma\right)^{(1)}+\tau^{2}\left(\Gamma_{\mu \nu} \gamma\right)^{(2)},
$$

where the first order term is

$$
\left(\Gamma_{\mu \nu}^{\gamma}\right)^{(1)}=\frac{1}{2}\left(\bar{\nabla}_{\mu} h_{\nu}^{\gamma}+\bar{\nabla}_{\nu} h_{\mu}^{\gamma}-\bar{\nabla}^{\gamma} h_{\mu \nu}\right),
$$

and the second order one is 


$$
\left(\Gamma_{\mu \nu} \gamma\right)^{(2)}=-h^{\gamma \delta}\left(\Gamma_{\mu \nu \delta}\right)^{(1)}
$$

Since it is a background tensor, we can raise and lower the indices with $\bar{g}_{\mu \nu}$

$$
\left(\Gamma_{\mu \nu \delta}\right)^{(1)}=\bar{g}_{\gamma \delta}\left(\Gamma_{\mu \nu} \gamma\right)^{(2)}
$$

The first order linearized Riemann tensor is

$$
\left(R_{\mu \sigma \nu}^{\rho}\right)^{(1)}=\bar{\nabla}_{\sigma}\left(\Gamma_{\nu \mu}^{\rho}\right)^{(1)}-\bar{\nabla}_{\nu}\left(\Gamma_{\sigma \mu} \rho\right)^{(1)},
$$

and the second order linearized Riemann tensor is

$$
\begin{aligned}
\left(R_{\mu \sigma \nu}^{\rho}\right)^{(2)}= & \bar{\nabla}_{\sigma}\left(\Gamma_{\nu \mu}{ }^{\rho}\right)^{(2)}-\bar{\nabla}_{\nu}\left(\Gamma_{\sigma \mu} \rho\right)^{(2)} \\
& -\left(\Gamma_{\mu \nu}{ }^{\alpha}\right)^{(1)}\left(\Gamma_{\sigma \alpha}{ }^{\rho}\right)^{(1)}+\left(\Gamma_{\mu \sigma}{ }^{\alpha}\right)^{(1)}\left(\Gamma_{\nu \alpha} \rho\right)^{(1)} .
\end{aligned}
$$

The first order linearized Ricci tensor is

$$
\left(R_{\mu \nu}\right)^{(1)}=\bar{\nabla}_{\sigma}\left(\Gamma_{\mu \nu} \sigma\right)^{(1)}-\bar{\nabla}_{\nu}\left(\Gamma_{\sigma \mu} \sigma\right)^{(1)}
$$

and the second order linearized Ricci tensor is

$$
\begin{aligned}
\left(R_{\mu \nu}\right)^{(2)}= & \bar{\nabla}_{\sigma}\left(\Gamma_{\nu \mu}{ }^{\sigma}\right)^{(2)}-\bar{\nabla}_{\nu}\left(\Gamma_{\sigma \mu}{ }^{\sigma}\right)^{(2)}-\left(\Gamma_{\mu \nu}{ }^{\alpha}\right)^{(1)}\left(\Gamma_{\sigma \alpha} \sigma\right)^{(1)} \\
& +\left(\Gamma_{\mu \sigma}{ }^{\alpha}\right)^{(1)}\left(\Gamma_{\nu \alpha} \sigma\right)^{(1)}
\end{aligned}
$$

We shall need the explicit form of it in terms of the $h_{\mu \nu}$ field which reads

$$
\begin{aligned}
\left(R_{\mu \nu}\right)^{(2)}= & -\frac{1}{2} \bar{\nabla}_{\rho}\left[h^{\rho \beta}\left(\bar{\nabla}_{\mu} h_{\nu \beta}+\bar{\nabla}_{\nu} h_{\mu \beta}-\bar{\nabla}_{\beta} h_{\nu \mu}\right)\right] \\
& +\frac{1}{2} \bar{\nabla}_{\nu}\left[h^{\rho \beta} \bar{\nabla}_{\mu} h_{\rho \beta}\right]+\frac{1}{4}\left(\bar{\nabla}_{\mu} h_{\rho \beta}\right) \bar{\nabla}_{\nu} h^{\rho \beta} \\
& +\frac{1}{4}\left(\bar{\nabla}^{\beta} h\right)\left(\bar{\nabla}_{\mu} h_{\nu \beta}+\bar{\nabla}_{\nu} h_{\mu \beta}-\bar{\nabla}_{\beta} h_{\nu \mu}\right) \\
& -\frac{1}{2}\left(\bar{\nabla}_{\beta} h_{\nu \alpha}\right) \bar{\nabla}^{\beta} h_{\mu}^{\alpha}+\frac{1}{2}\left(\bar{\nabla}_{\beta} h_{\nu \alpha}\right) \bar{\nabla}^{\alpha} h_{\mu}^{\beta} .
\end{aligned}
$$

The linearized scalar curvature is

$$
(R)^{(1)}=\bar{\nabla}_{\alpha} \bar{\nabla}_{\beta} h^{\alpha \beta}-\bar{\square} h-\bar{R}_{\mu \nu} h^{\mu \nu},
$$

and the second order linearized scalar curvature is

$$
(R)^{(2)}=\bar{R}_{\mu \nu} h_{\alpha}^{\mu} h^{\alpha \nu}-\left(R_{\mu \nu}\right)^{(1)} h^{\mu \nu}+\bar{g}^{\mu \nu}\left(R_{\mu \nu}\right)^{(2)} .
$$

Explicitly we have

$$
\begin{aligned}
(R)^{(2)}= & -\frac{1}{2} \bar{\nabla}_{\rho}\left[h^{\rho \beta}\left(2 \bar{\nabla}_{\sigma} h_{\beta}^{\sigma}-\bar{\nabla}_{\beta} h\right)\right]+\frac{1}{2} \bar{\nabla}_{\sigma}\left[h^{\rho \beta} \bar{\nabla}_{\sigma} h_{\rho \beta}\right] \\
& +\frac{1}{4}\left(\bar{\nabla}^{\beta} h\right)\left(2 \bar{\nabla}_{\sigma} h_{\beta}^{\sigma}-\bar{\nabla}_{\beta} h\right) \\
& -\frac{1}{4}\left(\bar{\nabla}_{\sigma} h_{\rho \beta}\right) \bar{\nabla}^{\sigma} h^{\rho \beta}+\frac{1}{2}\left(\bar{\nabla}_{\sigma} h_{\rho \beta}\right) \bar{\nabla}^{\rho} h^{\sigma \beta} \\
& -\frac{1}{2} h^{\rho \beta}\left[2 \bar{\nabla}_{\sigma} \bar{\nabla}_{\rho} h_{\beta}^{\sigma}-\square h_{\rho \beta}-\bar{\nabla}_{\rho} \bar{\nabla}_{\beta} h\right]+\bar{R}_{\rho \beta} h^{\rho \alpha} h_{\alpha}^{\beta} .
\end{aligned}
$$

Using the above results, let us find how the second order linearized form of the Einstein tensor transform under the gauge transformations generated by the flow of $X$. In the index-free notation one has

$$
\begin{aligned}
& D^{2} \operatorname{Ein}\left({ }^{(4)} g\right) \cdot\left({ }^{(4)} h, \mathcal{L}_{(4) X}{ }^{(4)} g\right)+D \operatorname{Ein}\left({ }^{(4)} g\right) \cdot \mathcal{L}_{(4) X}{ }^{(4)} h \\
& \quad=\mathcal{L}_{(4) X}\left(D \operatorname{Ein}\left({ }^{(4)} g\right) \cdot{ }^{(4)} h\right),
\end{aligned}
$$

which reads in local coordinates as

$\delta_{X}\left(G_{\mu \nu}\right)^{(2)} \cdot[h, h]+\left(G_{\mu \nu}\right)^{(1)} \cdot \mathcal{L}_{X} h=\mathcal{L}_{X}\left(G_{\mu \nu}\right)^{(1)} \cdot h$.

Let us prove this. By definition we have

$$
\begin{aligned}
\delta_{X}\left(G_{\mu \nu}\right)^{(2)} \cdot[h, h]= & \delta_{X}\left(R_{\mu \nu}\right)^{(2)} \cdot[h, h]-\frac{1}{2} \bar{g}_{\mu \nu} \delta_{X}(R)^{(2)} \cdot[h, h] \\
& -\frac{1}{2}(R)^{(1)} \cdot h \delta_{X} h_{\mu \nu}-\frac{1}{2} h_{\mu \nu} \delta_{X}(R)^{(1)} \cdot h .
\end{aligned}
$$

Let us calculate the right-hand side of the equation term by term.

$$
\begin{aligned}
\delta_{X}\left(R_{\mu \nu}\right)^{(2)} \cdot[h, h]= & -\left(\delta_{X} h^{\rho \beta}\right)\left(\bar{\nabla}_{\rho}\left(\Gamma_{\nu \mu \beta}\right)^{(1)}-\bar{\nabla}_{\nu}\left(\Gamma_{\rho \mu \beta}\right)^{(1)}\right) \\
& -h^{\rho \beta} \delta_{X}\left(\bar{\nabla}_{\rho}\left(\Gamma_{\nu \mu \beta}\right)^{(1)}-\bar{\nabla}_{\nu}\left(\Gamma_{\rho \mu \beta}\right)^{(1)}\right) \\
& -\delta_{X}\left(\left(\Gamma_{\mu \nu}{ }^{\alpha}\right)^{(1)}\left(\Gamma_{\sigma \alpha}{ }^{\sigma}\right)^{(1)}\right. \\
& \left.-\left(\Gamma_{\mu \sigma}{ }^{\alpha}\right)^{(1)}\left(\Gamma_{\nu}{ }^{\sigma}{ }_{\alpha}\right)^{(1)}\right) .
\end{aligned}
$$

Since one has

$$
\delta_{X} h^{\rho \beta}=-\mathcal{L}_{X} \bar{g}^{\rho \beta},
$$

using the identities (A4), (A5) we have

$$
\begin{aligned}
\delta_{X}\left(R_{\mu \nu}\right)^{(2)} \cdot[h, h]= & \mathcal{L}_{X}\left(R_{\mu \nu}\right)^{(1)} \cdot h-\frac{1}{2} \bar{g}^{\rho \beta}\left[\overline { \nabla } _ { \rho } \mathcal { L } _ { X } \left(\bar{\nabla}_{\nu} h_{\mu \beta}\right.\right. \\
& \left.\left.+\bar{\nabla}_{\mu} h_{\nu \beta}-\bar{\nabla}_{\beta} h_{\mu \nu}\right)-\bar{\nabla}_{\nu} \mathcal{L}_{X} \bar{\nabla}_{\mu} h_{\rho \beta}\right] \\
& +\left(\bar{\nabla}_{\nu} h_{\sigma}{ }^{\rho}\right) \delta_{X}\left(\Gamma_{\rho \mu}{ }^{\sigma}\right)^{(1)} \\
& -\left(\bar{\nabla}_{\rho} h_{\sigma}{ }^{\rho}\right) \delta_{X}\left(\Gamma_{\nu \mu}{ }^{\sigma}\right)^{(1)} \\
& -h^{\rho}{ }_{\beta} \delta_{X}\left(R^{\beta}{ }_{\mu \rho \nu}\right)^{(1)} \cdot h .
\end{aligned}
$$

Finally one can find 
$\delta_{X}\left(R_{\mu \nu}\right)^{(2)} \cdot[h, h]=\mathcal{L}_{X}\left(R_{\mu \nu}\right)^{(1)} \cdot h-\left(R_{\mu \nu}\right)^{(1)} \cdot \mathcal{L}_{X} h$,

and from the following definition

$$
\begin{aligned}
(R)^{(2)} \cdot[h, h]= & \bar{R}_{\rho \sigma} h^{\sigma \lambda} h_{\lambda}{ }^{\rho}-h^{\sigma \rho}\left(R_{\rho \sigma}\right)^{(1)} \cdot h \\
& +\bar{g}^{\sigma \lambda}\left(R_{\rho \sigma}\right)^{(2)} \cdot[h, h],
\end{aligned}
$$

one can find

$$
\begin{aligned}
\delta_{X}(R)^{(2)} \cdot[h, h]= & \mathcal{L}_{X}(R)^{(1)} \cdot h \\
& -\left[\bar{g}^{\sigma \rho}\left(R_{\rho \sigma}\right)^{(1)} \cdot \mathcal{L}_{X} h-\bar{R}^{\rho \sigma} \mathcal{L}_{X} h_{\sigma \rho}\right],
\end{aligned}
$$

which can be reduced to

$$
\delta_{X}(R)^{(2)} \cdot[h, h]=\mathcal{L}_{X}(R)^{(1)} \cdot h-(R)^{(1)} \cdot \mathcal{L}_{X} h .
$$

Then we can collect these to get the gauge transformation of the second order expansion of the Einstein tensor as

$$
\begin{aligned}
\delta_{X}\left(G_{\mu \nu}\right)^{(2)} \cdot[h, h] & \\
= & \mathcal{L}_{X}\left[\left(R_{\mu \nu}\right)^{(1)} \cdot h-\frac{1}{2} \bar{g}_{\mu \nu}(R)^{(1)} \cdot h-\frac{1}{2} h_{\mu \nu} \bar{R}\right] \\
& -\left[\left(R_{\mu \nu}\right)^{(1)} \cdot \mathcal{L}_{X} h-\frac{1}{2} \bar{g}_{\mu \nu}(R)^{(1)} \cdot \mathcal{L}_{X} h-\frac{1}{2} \bar{R} \mathcal{L}_{X} h_{\mu \nu}\right] .
\end{aligned}
$$

The first line is the Lie derivative of the linearized Einstein tensor and the second line is the linearized Einstein tensor evaluated at $\mathcal{L}_{X} h$.

$\delta_{X}\left(G_{\mu \nu}\right)^{(2)} \cdot[h, h]=\mathcal{L}_{X}\left(G_{\mu \nu}\right)^{(1)} \cdot h-\left(G_{\mu \nu}\right)^{(1)} \cdot \mathcal{L}_{X} h$,

which is the desired formula.

\section{APPENDIX B: EXPLICIT FORM OF THE $K_{\mu \nu}$ TENSOR IN ADS}

Here let us depict some of the intermediate steps leading to (32). Assuming a general form for the $k_{\mu \nu}$ as

$$
k_{\mu \nu}=a h_{\mu \beta} h_{\nu}^{\beta}+b h h_{\mu \nu}+\bar{g}_{\mu \nu}\left(c h_{\alpha \beta}^{2}+d h^{2}\right) .
$$

The first order Ricci operator evaluated at $k$ is

$$
\left(R_{\mu \nu}\right)^{(1)} \cdot k=\frac{1}{2}\left(\bar{\nabla}_{\alpha} \bar{\nabla}_{\mu} k_{\nu}^{\alpha}+\bar{\nabla}_{\alpha} \bar{\nabla}_{\nu} k_{\mu}^{\alpha}-\square k_{\mu \nu}-\bar{\nabla}_{\mu} \bar{\nabla}_{\nu} k\right),
$$

whose explicit form follows as

$$
\begin{aligned}
\left(R_{\mu \nu}\right)^{(1)} \cdot k= & \frac{a}{2}\left(\bar{\nabla}_{\alpha} \bar{\nabla}_{\mu} h^{\alpha \beta} h_{\beta \nu}+\bar{\nabla}_{\alpha} \bar{\nabla}_{\nu} h^{\alpha \beta} h_{\beta \mu}-\square h_{\nu}^{\beta} h_{\beta \mu}-\bar{\nabla}_{\mu} \bar{\nabla}_{\nu} h_{\alpha \beta}^{2}\right)+\frac{b}{2}\left(\bar{\nabla}_{\alpha} \bar{\nabla}_{\mu} h h_{\nu}^{\alpha}+\bar{\nabla}_{\alpha} \bar{\nabla}_{\nu} h h_{\mu}^{\alpha}-\square h h_{\mu \nu}-\bar{\nabla}_{\mu} \bar{\nabla}_{\nu} h^{2}\right) \\
& +\frac{c}{2}\left(\bar{\nabla}_{\nu} \bar{\nabla}_{\mu} h_{\alpha \beta}^{2}+\bar{\nabla}_{\mu} \bar{\nabla}_{\nu} h_{\alpha \beta}^{2}-\bar{g}_{\mu \nu} \square h_{\alpha \beta}^{2}-D \bar{\nabla}_{\mu} \bar{\nabla}_{\nu} h_{\alpha \beta}^{2}\right)+\frac{d}{2}\left(\bar{\nabla}_{\nu} \bar{\nabla}_{\mu} h^{2}+\bar{\nabla}_{\mu} \bar{\nabla}_{\nu} h^{2}-\bar{g}_{\mu \nu} \square h^{2}-D \bar{\nabla}_{\mu} \bar{\nabla}_{\nu} h^{2}\right) .
\end{aligned}
$$

We should set $a=1$ and $b=-1 / 2$ to get the second order linearized Ricci tensor

$$
\begin{aligned}
\left(R_{\mu \nu}\right)^{(1)} \cdot k= & \bar{\nabla}_{\alpha}\left(h^{\alpha \beta}\left(\Gamma_{\mu \nu \beta}\right)^{(1)}\right)-\bar{\nabla}_{\nu}\left(h^{\alpha \beta}\left(\Gamma_{\mu \alpha \beta}\right)^{(1)}\right)-\left(\Gamma_{\beta}^{\alpha \beta}\right)^{(1)}\left(\Gamma_{\mu \nu \alpha}\right)^{(1)} \\
& +\frac{1}{2} \bar{\nabla}_{\alpha}\left(h_{\beta \nu} \bar{\nabla}_{\mu} h^{\alpha \beta}+h_{\beta \mu} \bar{\nabla}_{\nu} h^{\alpha \beta}+h^{\alpha \beta} \bar{\nabla}_{\beta} h_{\nu \mu}-\bar{\nabla}^{\alpha}\left(h_{\nu}^{\beta} h_{\beta \mu}\right)\right)-\frac{1}{2} \bar{\nabla}_{\nu}\left(h^{\alpha \beta} \bar{\nabla}_{\mu} h_{\alpha \beta}\right) \\
& -\frac{1}{4} h \bar{\nabla}_{\alpha}\left(\bar{\nabla}_{\mu} h_{\nu}^{\alpha}+\bar{\nabla}_{\nu} h_{\mu}^{\alpha}-\bar{\nabla}^{\alpha} h_{\mu \nu}\right)+\frac{1}{2} \bar{\nabla}_{\nu}\left(h \bar{\nabla}_{\mu} h\right)-\frac{1}{4} \bar{\nabla}_{\alpha}\left(h_{\nu}^{\alpha} \bar{\nabla}_{\mu} h+h_{\mu}^{\alpha} \bar{\nabla}_{\nu} h-h_{\mu \nu} \bar{\nabla}^{\alpha} h\right) \\
& +\frac{c}{2}\left((2-D) \bar{\nabla}_{\nu} \bar{\nabla}_{\mu} h_{\alpha \beta}^{2}-\bar{g}_{\mu \nu} \bar{\square} h_{\alpha \beta}^{2}\right)+\frac{d}{2}\left((2-D) \bar{\nabla}_{\nu} \bar{\nabla}_{\mu} h^{2}-\bar{g}_{\mu \nu} \bar{\square} h^{2}\right) .
\end{aligned}
$$

Finally the Ricci tensor evaluated at $k$ becomes

$$
\begin{aligned}
\left(R_{\mu \nu}\right)^{(1)} \cdot k= & -\left(R_{\mu \nu}\right)^{(2)} \cdot[h, h]-\frac{3}{4} \bar{\nabla}_{\nu} h^{\alpha \beta} \bar{\nabla}_{\mu} h_{\alpha \beta}+\frac{1}{2} \bar{\nabla}_{\alpha} h_{\mu \beta} \bar{\nabla}^{\alpha} h_{\nu}^{\beta}-\frac{1}{2} \bar{\nabla}_{\alpha} h_{\mu \beta} \bar{\nabla}^{\beta} h_{\nu}^{\alpha} \\
& +\frac{1}{2} \bar{\nabla}_{\alpha}\left(h_{\beta \nu} \bar{\nabla}_{\mu} h^{\alpha \beta}+h_{\beta \mu} \bar{\nabla}_{\nu} h^{\alpha \beta}+h^{\alpha \beta} \bar{\nabla}_{\beta} h_{\nu \mu}-\bar{\nabla}^{\alpha}\left(h_{\nu}^{\beta} h_{\beta \mu}\right)\right)-\frac{1}{2} h^{\alpha \beta} \bar{\nabla}_{\nu} \bar{\nabla}_{\mu} h_{\alpha \beta}-\frac{h}{2}\left(R_{\mu \nu}\right)^{(1)} \cdot h-\frac{1}{4} h \bar{\nabla}_{\nu} \bar{\nabla}_{\mu} h \\
& +\frac{1}{2} \bar{\nabla}_{\nu}\left(h \bar{\nabla}_{\mu} h\right)-\frac{1}{4} \bar{\nabla}_{\alpha}\left(h_{\nu}^{\alpha} \bar{\nabla}_{\mu} h+h_{\mu}^{\alpha} \bar{\nabla}_{\nu} h-h_{\mu \nu} \bar{\nabla}^{\alpha} h\right) \\
& +\frac{c}{2}\left((2-D) \bar{\nabla}_{\nu} \bar{\nabla}_{\mu} h_{\alpha \beta}^{2}-\bar{g}_{\mu \nu} \bar{\square} h_{\alpha \beta}^{2}\right)+\frac{d}{2}\left((2-D) \bar{\nabla}_{\nu} \bar{\nabla}_{\mu} h^{2}-\bar{g}_{\mu \nu} \bar{\square} h^{2}\right),
\end{aligned}
$$


from which one can find the scalar curvature

$$
\begin{aligned}
(R)^{(1)} \cdot k= & -(R)^{(2)} \cdot[h, h]-\frac{5}{4} \bar{\nabla}^{\mu} h^{\alpha \beta} \bar{\nabla}_{\mu} h_{\alpha \beta}+\frac{1}{2} \bar{\nabla}_{\alpha} h_{\mu \beta} \bar{\nabla}^{\beta} h^{\mu \alpha}+\frac{1}{2} h^{\alpha \beta} \bar{\nabla}_{\alpha} \bar{\nabla}_{\beta} h-h^{\alpha \beta} \bar{\square} h_{\alpha \beta}-\frac{h}{2}(R)^{(1)} \cdot h+\frac{1}{2} h \bar{\square} h \\
& \left.+\frac{3}{4} \bar{\nabla}^{\mu} h \bar{\nabla}_{\mu} h+c(1-D) \bar{\square} h_{\alpha \beta}^{2}+d(1-D) \bar{\square} h^{2}\right)-\bar{R}\left(c h_{\alpha \beta}^{2}+d h^{2}\right) .
\end{aligned}
$$

Finally the linearized Einstein tensor can be found as

$$
\begin{aligned}
\left(\mathcal{G}_{\mu \nu}\right)^{(1)} \cdot k= & -\left(\mathcal{G}_{\mu \nu}\right)^{(2)} \cdot[h, h]-\frac{1}{2} h_{\mu \nu}(R)^{(1)} \cdot h-\frac{h}{2}\left(\mathcal{G}_{\mu \nu}\right)^{(1)} \cdot h-\frac{3}{4} \bar{\nabla}_{\nu} h^{\alpha \beta} \bar{\nabla}_{\mu} h_{\alpha \beta}+\frac{1}{2} \bar{\nabla}_{\alpha} h_{\mu \beta} \bar{\nabla}^{\alpha} h_{\nu}^{\beta}-\frac{1}{2} \bar{\nabla}_{\alpha} h_{\mu \beta} \bar{\nabla}^{\beta} h_{\nu}^{\alpha} \\
& +\frac{1}{2} \bar{\nabla}_{\alpha}\left(h_{\beta \nu} \bar{\nabla}_{\mu} h^{\alpha \beta}+h_{\beta \mu} \bar{\nabla}_{\nu} h^{\alpha \beta}+h^{\alpha \beta} \bar{\nabla}_{\beta} h_{\nu \mu}-\bar{\nabla}^{\alpha}\left(h_{\nu}^{\beta} h_{\beta \mu}\right)\right)-\frac{1}{2} h^{\alpha \beta} \bar{\nabla}_{\nu} \bar{\nabla}_{\mu} h_{\alpha \beta}-\frac{1}{4} h \bar{\nabla}_{\nu} \bar{\nabla}_{\mu} h+\frac{1}{2} \bar{\nabla}_{\nu}\left(h \bar{\nabla}_{\mu} h\right) \\
& -\frac{1}{4} \bar{\nabla}_{\alpha}\left(h_{\nu}^{\alpha} \bar{\nabla}_{\mu} h+h_{\mu}^{\alpha} \bar{\nabla}_{\nu} h-h_{\mu \nu} \bar{\nabla}^{\alpha} h\right)+\frac{c}{2}(2-D) \bar{\nabla}_{\nu} \bar{\nabla}_{\mu} h_{\alpha \beta}^{2}+\frac{d}{2}(2-D) \bar{\nabla}_{\nu} \bar{\nabla}_{\mu} h^{2} \\
& -\frac{1}{2} \bar{g}_{\mu \nu}\left[-\frac{5}{4} \bar{\nabla}^{\sigma} h^{\alpha \beta} \bar{\nabla}_{\sigma} h_{\alpha \beta}+\frac{1}{2} \bar{\nabla}_{\alpha} h_{\sigma \beta} \bar{\nabla}^{\beta} h^{\sigma \alpha}\right]-\frac{1}{2} \bar{g}_{\mu \nu}\left[\frac{1}{2} h^{\alpha \beta} \bar{\nabla}_{\alpha} \bar{\nabla}_{\beta} h-h^{\alpha \beta} \bar{\square} h_{\alpha \beta}+\frac{1}{2} h \bar{\square} h+\frac{3}{4} \bar{\nabla}^{\sigma} h \bar{\nabla}_{\sigma} h\right] \\
& \left.-\frac{1}{2} \bar{g}_{\mu \nu}\left[c(2-D) \bar{\square} h_{\alpha \beta}^{2}+d(2-D) \bar{\square} h^{2}\right)-\bar{R}\left(c h_{\alpha \beta}^{2}+d h^{2}\right)\right]-\frac{2 \Lambda}{D-2}\left[h_{\mu \beta} h_{\nu}^{\beta}+\bar{g}_{\mu \nu}\left(c h_{\alpha \beta}^{2}+d h^{2}\right)\right] .
\end{aligned}
$$

Using these, one can find the final form of the $K_{\mu \nu}$ tensor as

$$
\begin{aligned}
K_{\mu \nu}= & -\frac{1}{2} h_{\mu \nu}(R)^{(1)} \cdot h-\frac{h}{2}\left(\mathcal{G}_{\mu \nu}\right)^{(1)} \cdot h-\frac{3}{4} \bar{\nabla}_{\nu} h^{\alpha \beta} \bar{\nabla}_{\mu} h_{\alpha \beta}+\frac{1}{2} \bar{\nabla}_{\alpha} h_{\mu \beta} \bar{\nabla}^{\alpha} h_{\nu}^{\beta}-\frac{1}{2} \bar{\nabla}_{\alpha} h_{\mu \beta} \bar{\nabla}^{\beta} h_{\nu}^{\alpha} \\
& +\frac{1}{2} \bar{\nabla}_{\alpha}\left(h_{\beta \nu} \bar{\nabla}_{\mu} h^{\alpha \beta}+h_{\beta \mu} \bar{\nabla}_{\nu} h^{\alpha \beta}+h^{\alpha \beta} \bar{\nabla}_{\beta} h_{\nu \mu}-\bar{\nabla}^{\alpha}\left(h_{\nu}^{\beta} h_{\beta \mu}\right)\right)-\frac{1}{2} h^{\alpha \beta} \bar{\nabla}_{\nu} \bar{\nabla}_{\mu} h_{\alpha \beta}-\frac{1}{4} h \bar{\nabla}_{\nu} \bar{\nabla}_{\mu} h+\frac{1}{2} \bar{\nabla}_{\nu}\left(h \bar{\nabla}_{\mu} h\right) \\
& -\frac{1}{4} \bar{\nabla}_{\alpha}\left(h_{\nu}^{\alpha} \bar{\nabla}_{\mu} h+h_{\mu}^{\alpha} \bar{\nabla}_{\nu} h-h_{\mu \nu} \bar{\nabla}^{\alpha} h\right)+\frac{c}{2}(2-D) \bar{\nabla}_{\nu} \bar{\nabla}_{\mu} h_{\alpha \beta}^{2}+\frac{d}{2}(2-D) \bar{\nabla}_{\nu} \bar{\nabla}_{\mu} h^{2}-\frac{2 \Lambda}{D-2}\left[h_{\mu \beta} h_{\nu}^{\beta}+\bar{g}_{\mu \nu}\left(c h_{\alpha \beta}^{2}+d h^{2}\right)\right] \\
& -\frac{1}{2} \bar{g}_{\mu \nu}\left[-\frac{5}{4} \bar{\nabla}^{\sigma} h^{\alpha \beta} \bar{\nabla}_{\sigma} h_{\alpha \beta}+\frac{1}{2} \bar{\nabla}_{\alpha} h_{\sigma \beta} \bar{\nabla}^{\beta} h^{\sigma \alpha}\right]-\frac{1}{2} \bar{g}_{\mu \nu}\left[+\frac{1}{2} h^{\alpha \beta} \bar{\nabla}_{\alpha} \bar{\nabla}_{\beta} h-h^{\alpha \beta} \bar{\square} h_{\alpha \beta}+\frac{1}{2} h \bar{\square} h+\frac{3}{4} \bar{\nabla}^{\sigma} h \bar{\nabla}_{\sigma} h\right] \\
& \left.-\frac{1}{2} \bar{g}_{\mu \nu}\left[c(2-D) \bar{\square} h_{\alpha \beta}^{2}+d(2-D) \bar{\square} h^{2}\right)-\bar{R}\left(c h_{\alpha \beta}^{2}+d h^{2}\right)\right],
\end{aligned}
$$

whose gauge-fixed version was given in the text.

[1] D. Brill and S. Deser, Instability of closed spaces in general relativity, Commun. Math. Phys. 32, 291 (1973).

[2] A. H. Taub, Variational Principles in General Relativity, edited by C. Cattanes, Lectures at the Centro Internazionale Matematico Estiud, Bressanone (Edizioni Cremonese, Rome, 1970), p. 206.

[3] J. Girbau and L. Bruna, Stability by Linearization of Einstein's Field Equation (Springer, New York, 2010).

[4] Y. Choquet-Bruhat, General Relativity and the Einstein Equations (Oxford University Press, New York, 2009).

[5] W. Li, W. Song, and A. Strominger, Chiral gravity in three dimensions, J. High Energy Phys. 04 (2008) 082.

[6] A. Maloney, W. Song, and A. Strominger, Chiral gravity, log gravity and extremal CFT, Phys. Rev. D 81, 064007 (2010).
[7] A. Strominger, A simple proof of the chiral gravity conjecture, arXiv:0808.0506.

[8] S. Carlip, Chiral topologically massive gravity and extremal B-F scalars, J. High Energy Phys. 09 (2009) 083.

[9] S. Deser, R. Jackiw, and S. Templeton, Topologically massive gauge theories, Ann. Phys. (N.Y.) 140, 372 (1982).

[10] D. Grumiller and N. Johansson, Instability in cosmological topologically massive gravity at the chiral point, J. High Energy Phys. 07 (2008) 134.

[11] J. D. Brown and M. Henneaux, Central charges in the canonical realization of asymptotic symmetries: An example from three-dimensional gravity, Commun. Math. Phys. 104, 207 (1986).

[12] H. Lu and C. N. Pope, Critical Gravity in Four Dimensions, Phys. Rev. Lett. 106, 181302 (2011). 
[13] S. Deser, H. Liu, H. Lu, C. N. Pope, T. C. Sisman, and B. Tekin, Critical points of D-dimensional extended gravities, Phys. Rev. D 83, 061502 (2011).

[14] S. Deser and Y. Choquet-Bruhat, On the stability of flat space, Ann. Phys. (N.Y.) 81, 165 (1973).

[15] A. E. Fischer and J. E. Marsden, Linearization stability of the Einstein equations, Bull. Am. Math. Soc., 79, 997 (1973).

[16] A. E. Fischer, J. E. Marsden, and V. Moncrief, The structure of the space of solutions of Einstein's equations. I. One Killing field, Ann. I. H. P.: Phys. Theor. 33, 147 (1980).

[17] J.E. Marsden, in Lectures on Geometric Methods in Mathematical Physics, CBMS- NSF Regional Conf. Ser. in Appl. Math. (SIAM, Philadelphia, Pa., 1981), Vol. 37.

[18] L. F. Abbott and S. Deser, Stability of gravity with a cosmological constant, Nucl. Phys. B195, 76 (1982).

[19] S. Deser and B. Tekin, Energy in generic higher curvature gravity theories, Phys. Rev. D 67, 084009 (2003); Gravitational Energy in Quadratic Curvature Gravities, Phys. Rev. Lett. 89, 101101 (2002).

[20] C. Senturk, T. C. Sisman, and B. Tekin, Energy and angular momentum in generic F(Riemann) theories, Phys. Rev. D 86, 124030 (2012).

[21] V. Moncrief, Spacetime symmetries and linearization stability of the Einstein equations. I, J. Math. Phys. (N.Y.) 16, 493 (1975).

[22] J. M. Arms and J.E. Marsden The absence of Killing fields is necessary for linearization stability of Einstein's equations, Indiana University Mathematics Journal 28, 119 (1979).

[23] A. Higuchi, Possible constraints on string theory in closed space with symmetries, arXiv:hep-th/0511237.

[24] D. G. Boulware, G. T. Horowitz, and A. Strominger, Zero Energy Theorem for Scale Invariant Gravity, Phys. Rev. Lett. 50, 1726 (1983).

[25] R. Schoen and S. T. Yau, On the proof of the positive mass conjecture in general relativity, Commun. Math. Phys. 65, 45 (1979); Proof of the positive mass theorem. II, Commun. Math. Phys. 79, 231 (1981).

[26] E. Witten, A simple proof of the positive energy theorem, Commun. Math. Phys. 80, 381 (1981).

[27] D. R. Brill and S. Deser, Variational methods and positive energy in general relativity, Ann. Phys. (N.Y.) 50, 548 (1968).

[28] S. Deser, D. R. Brill, and L. D. Faddeev, Sign of gravitational energy, Phys. Lett. A 26, 538 (1968).

[29] R. Arnowitt, S. Deser, and C. W. Misner, Canonical variables for general relativity, Phys. Rev. 117, 1595 (1960); The dynamics of general relativity, Gen. Relativ. Gravit. 40, 1997 (2008).

[30] B. Tekin, Particle content of quadratic and $f\left(R_{\mu \nu \sigma \rho}\right)$ theories in $(A) d S$, Phys. Rev. D 93, 101502 (2016).
[31] M. Alishahiha and R. Fareghbal, $D$-dimensional log gravity, Phys. Rev. D 83, 084052 (2011).

[32] I. Gullu, M. Gurses, T. C. Sisman, and B. Tekin, AdS waves as exact solutions to quadratic gravity, Phys. Rev. D 83, 084015 (2011).

[33] J. M. Maldacena, The large $\mathrm{N}$ limit of superconformal field theories and supergravity, Int. J. Theor. Phys. 38, 1113 (1999) [The large $\mathrm{N}$ limit of superconformal field theories and supergravityAdv. Theor. Math. Phys. 2, 231 (1998)].

[34] S. Deser and B. Tekin, Energy in topologically massive gravity, Classical Quantum Gravity 20, L259 (2003).

[35] S. Olmez, O. Sarioglu, and B. Tekin, Mass and angular momentum of asymptotically ads or flat solutions in the topologically massive gravity, Classical Quantum Gravity 22, 4355 (2005).

[36] S. Carlip, S. Deser, A. Waldron, and D. K. Wise, Cosmological topologically massive gravitons and photons, Classical Quantum Gravity 26, 075008 (2009).

[37] G. Giribet, M. Kleban, and M. Porrati, Topologically massive gravity at the chiral point is not chiral, J. High Energy Phys. 10 (2008) 045.

[38] D. Grumiller, W. Riedler, J. Rosseel, and T. Zojer, Holographic applications of logarithmic conformal field theories, J. Phys. A 46, 494002 (2013).

[39] A. N. Petrov, S. M. Kopeikin, R. R. Lompay, and B. Tekin, Metric theories of gravity: Perturbations and conservation laws, De Gruyter Stud. Math. Phys. (De Gruyter, Berlin, 2017), Vol. 38.

[40] S. Deser and B. Tekin, New energy definition for higher curvature gravities, Phys. Rev. D 75, 084032 (2007).

[41] E. Sezgin and Y. Tanii, Witten-Nester energy in topologically massive gravity, Classical Quantum Gravity 26, 235005 (2009).

[42] E. Bergshoeff, O. Hohm, W. Merbis, A. J. Routh, and P. K. Townsend, Minimal massive 3D gravity, Classical Quantum Gravity 31, 145008 (2014).

[43] G. Alkac, L. Basanisi, E. Kilicarslan, and B. Tekin, Unitarity problems in $3 D$ gravity theories, Phys. Rev. D 96, 024010 (2017).

[44] B. Tekin, Minimal massive gravity: Conserved charges, excitations and the chiral gravity limit, Phys. Rev. D 90, 081701 (2014).

[45] M. Alishahiha, M. M. Qaemmaqami, A. Naseh, and A. Shirzad, On 3D minimal massive gravity, J. High Energy Phys. 12 (2014) 033.

[46] R. Gicquaud, Linearization stability of the Einstein constraint equations on an asymptotically hyperbolic manifold, J. Math. Phys. (N.Y.) 51, 072501 (2010).

[47] I. Gullu, T. C. Sisman, and B. Tekin, Unitarity analysis of general Born-Infeld gravity theories, Phys. Rev. D 82, 124023 (2010). 